Publications of the Astronomical Society of the Pacific, 125:1031-1055, 2013 September

(C) 2013. The Astronomical Society of the Pacific. All rights reserved. Printed in U.S.A.

\title{
Las Cumbres Observatory Global Telescope Network
}

\author{
T. M. Brown, N. Baliber, ${ }^{1}$ F. B. Bianco, ${ }^{2}$ M. Bowman, B. Burleson, P. Conway, M. Crellin, É. Depagne, ${ }^{3}$ \\ J. De Vera, B. Dilday, D. Dragomir, M. Dubberley, ${ }^{4}$ J. D. Eastman, M. Elphick, M. Falarski, S. Foale, \\ M. Ford, B. J. Fulton, ${ }^{5}$ J. Garza, E. L. Gomez, M. Graham, R. Greene, B. Haldeman, E. Hawkins, \\ B. Haworth, R. Haynes, M. Hidas, A. E. Huelstrom, D. A. Howell, J. Hygelund, T. A. Lister, \\ R. Lobdill, J. Martinez, D. S. Mullins, M. Norbury, J. Parrent, R. Paulson, D. L. Petry, \\ A. Pickles, V. Posner, W. E. Rosing, R. Ross, D. J. Sand, ${ }^{6}$ E. S. Saunders, J. Shobbrook, \\ A. Shrorer, ${ }^{7}$ R. A. Street, D. Thomas, Y. Tsapras, J. R. Tufts, S. Valenti, \\ K. VAnder Horst, Z. Walker, G. White, AND M. Willis \\ Las Cumbres Observatory Global Telescope Network, 6740 Cortona Dr. Suite 102, Goleta, CA 93117 \\ Received 2013 May 03; accepted 2013 July 11; published 2013 September 10
}

\begin{abstract}
Las Cumbres Observatory Global Telescope (LCOGT) is a young organization dedicated to timedomain observations at optical and (potentially) near-IR wavelengths. To this end, LCOGT is constructing a worldwide network of telescopes, including the two $2 \mathrm{~m}$ Faulkes telescopes, as many as $17 \times 1 \mathrm{~m}$ telescopes, and as many as $23 \times 40 \mathrm{~cm}$ telescopes. These telescopes initially will be outfitted for imaging and (excepting the $40 \mathrm{~cm}$ telescopes) spectroscopy at wavelengths between the atmospheric UV cutoff and the roughly 1- $\mu$ m limit of silicon detectors. Since the first of LCOGT's $1 \mathrm{~m}$ telescopes are now being deployed, we lay out here LCOGT's scientific goals and the requirements that these goals place on network architecture and performance, we summarize the network's present and projected level of development, and we describe our expected schedule for completing it. In the bulk of the paper, we describe in detail the technical approaches that we have adopted to attain desired performance. In particular, we discuss our choices for the number and location of network sites, for the number and sizes of telescopes, for the specifications of the first generation of instruments, for the software that will schedule and control the network's telescopes and reduce and archive its data, and for the structure of the scientific and educational programs for which the network will provide observations.
\end{abstract}

\section{INTRODUCTION}

For about the last century, the dominant line of development in astronomical facilities has been to build larger and larger telescopes to observe fainter and fainter objects. This approach has been spectacularly successful, but the cost of large boundarypushing telescopes has grown to the point that obtaining funding for them may soon become impractical. Thus, it is desirable to look for observing strategies that permit less expensive ways to learn about the cosmos. One such approach goes under the rubric of "time-domain astronomy"; the idea is to exploit the temporal variability of astronomical sources of radiation to learn something about their physical structures and their interactions

${ }^{1}$ Department of Astronomy, California Institute of Technology, Pasadena, CA 91125-0001.

${ }^{2}$ Department of Physics, New York University, New York, NY 10012.

${ }^{3}$ Leibniz-Institut für Astrophysik, Potsdam, Germany.

${ }^{4}$ Santa Barbara Infrared, Inc., Santa Barbara, CA.

${ }^{5}$ Institute for Astronomy, University of Hawaii, Honolulu, HI 96822.

${ }^{6}$ Physics Department, Texas Tech University, Lubbock, TX 79409-1051.

${ }^{7}$ Division of Geological and Planetary Sciences, California Institute of Technology, Pasadena, CA 91125. with other objects. Of course, time-domain astronomy is not new. Indeed, in the sense of positional astronomy (e.g., planetary motions), such studies go back to antiquity. But in the modern era, scheduling practices have not been conducive to observing programs that require (say) weeks of continuous observation of a single target, or near-instant response to triggers from short-lived transient phenomena, or even relatively sparse time coverage of every member of a large list of targets. With great effort, workers have arranged campaigns to monitor shortperiod variability of stars over long time spans (e.g., the Whole Earth Telescope, Nather et al. [1990], or the SONG network, now in construction, for asteroseismology and microlensing, Grundahl et al. [2008]), and by building special-purpose small telescopes, e.g., the ETC, LOTIS, ROTSE, and RAPTOR systems, to observe gamma-ray bursts (Vanderspek et al. 1992; Park et al. 1998; Akerlof et al. 2000; Vestrand et al. 2002) and PAIRITEL (Bloom et al. 2006), which in addition performs near-infrared observations of many other kinds of phenomena. But such projects are hampered by the lack of flexible facilities that can provide time-sampled data over a range of temporal cadences, dataset durations, and observing modes in a routine and systematic way. Las Cumbres Observatory Global 
Telescope (LCOGT) is such a facility. It is the first generalpurpose, flexibly-scheduled, multi-instrument optical observatory designed expressly to pursue astronomical research in the time domain. Its success depends not so much on advancing the state of the art in telescope technology as on deploying a global network of telescopes that exploits all the communication, coordination, automation, and data-processing strategies made possible by modern computing networks. The combination of robotic telescopes with internet communication proves to be a potent one, and one that should bring major advances in some branches of astronomical observing.

Here we describe LCOGT's aims and the hardware and software systems that we have built to meet them. The remainder of the paper is organized as follows. Section 2 gives a very brief description of the scientific opportunities offered by timedomain astronomy. Section 3 describes the general structurenumber and geographic distribution of sites, number and size of telescopes - that we believe necessary for a network of groundbased telescopes to exploit these opportunities. In $\S 4$ we describe the telescopes that we have deployed, or will soon deploy, to various nodes of the LCOGT network. We place particular emphasis on our network of $1 \mathrm{~m}$ telescopes, because they represent the most novel part of the growing network, and because they provide the largest portion of the network's observing capability. Section 5 describes the design and capabilities of the instruments (imaging and spectrographic) that are now installed on the network telescopes. Section 6 is a brief description of the distributed software system that schedules and assigns observing tasks to particular telescopes, performs initial reductions both for real-time quality assurance and as input for later scientific interpretation, and archives the result for access by users of the LCOGT Network (henceforth, "the Network") and by the public. We note that the Network's most novel and distinctive feature is the software system that organizes its functions. In this paper we describe this system at a high functional level; much more detailed descriptions of its major components may be found in Hawkins et al. (2010), and in Pickles et al. (2010, 2012). Section 7 describes our experience with shipping telescopes to their intended sites and bringing them through the process of deployment, start-up, and commissioning. In $\S 8$ we outline the goals and some of the methods and accomplishments of LCOGT's Education Program, which is built around ready access to the observing resources of the Network. Section 9 displays the first scientific result from our first remote Network $1 \mathrm{~m}$ telescope, obtained within weeks of its initial installation at the McDonald Observatory in Texas in 2012 April, and $\S 10$ gives a brief summary and describes prospects for the future.

\section{ASTRONOMY IN THE TIME DOMAIN}

From millisecond pulsars to binaries with periods of hundreds of years, astrophysical transients vary on timescales that span many orders of magnitude. Transient apparent brightnesses also range from naked-eye to well beyond the detection limit of current telescopes. Even if restricted to optical light, no one facility could be built to study all such phenomena. In this section we detail the characteristics of some of the most scientifically interesting transients and variable sources, which we used to optimize the design of a global network for studying time domain astronomy.

1. Dense time series: Planetary transits of a star and stellar variability have timescales of minutes to hours, requiring dense time-series monitoring (Charbonneau et al. 2000). High signalto-noise ratio $(\mathrm{S} / \mathrm{N})$ data are necessary to recover sub- $1 \%$ changes in brightness. But because detection and follow-up methods for such objects are biased towards bright stars, the bulk of known targets are fairly bright, often brighter than 12th magnitude. For these, moderate-aperture telescopes provide the requisite photometric precision. Continuous observations are also important to prevent aliasing when periodograms are constructed. This is problematic at single-site facilities, where a given target can normally be observed for 10 or fewer hours out of each 24 .

2. Episodic time series: Most supernovae rise and fall on a timescale of months. Many are powered by the radioactive decay of ${ }^{56} \mathrm{Ni}$ to ${ }^{56} \mathrm{Co}$ (half-life 6.1 days) and ${ }^{56} \mathrm{Co}$ to ${ }^{56} \mathrm{Fe}$ (halflife 77 days), so observations are generally required only every few days for the reconstruction of a lightcurve. The exception is at early times, e. g., catching the shock breakout from a red giant, which happens in hours. Just after explosion, the SN photosphere is also expanding and doubling on a similar timescale. It is now rare to catch a SN explosion so early (e.g., SN2012fe in M101), but with improved monitoring this will happen more frequently in the future. In such cases, it is necessary to observe a supernova as soon after discovery as is feasible, which is greatly facilitated by the longitude distribution of a global network. And because supernovae are sometimes used as distance indicators, precise multi-band absolute photometry is often required. Since they are always located in distant galaxies, the targets are rarely brighter than 14th magnitude. Therefore, substantial observing time is required to build supernova lightcurves, favoring a network composed of many nodes.

3. Triggered dense time series: Microlensing of stars by planets presents a unique challenge-a huge number of stars along a densely populated line of sight must be monitored for signs of variability, and when an anomalous microlens event is detected, dense time sampling with a cadence of minutes must be activated. A large number of telescopes distributed in longitude would ensure that observations are possible in the narrow time window when an anomaly is active.

4. Gamma-ray bursts: The optical counterparts of gamma-ray bursts (GRBs) fade as a power law on timescales from minutes to hours (Gehrels et al. 2009). Departures from power-law behavior (flares or breaks) are also observed, and carry information about such explosion parameters as the jet opening angle. Therefore, the most effective way to study bursts is to have a network of automated telescopes with a rapid response time and similar photometric response, well-distributed in longitude. 
Since GRBs are at cosmological distances, telescope apertures larger than $1 \mathrm{~m}$ are preferable.

5. Very rapid time series: Solar system bodies (e.g., Kuiper belt objects) may take only fractions of a second to transit a star (e. g., Elliot et al. 2010). Characterizing their size requires multi$\mathrm{Hz}$ observations at multiple sites to probe multiple chords across the occulting body.

6. Lucky imaging: Fast photometry cameras also enable "lucky" imaging, where many images per second are obtained, and only the $\sim 1 \%$ least affected by seeing are used to resolve small angles (Law et al. 2006).

7. Astrometry: Near-Earth Objects (NEOs) are routinely discovered by large surveys, but require targeted follow-up within days, to provide astrometric data for accurate orbital elements before the object is lost to view. Each of many targets require only small amounts of time, making such work ideal for a robotic facility.

8. Classification spectra of supernovae: Thousands of supernovae are discovered per year, but spectroscopic observations must be done to determine redshifts and to classify them. Since these extragalactic sources can be faint, a high-efficiency spectrograph on a large-aperture telescope is desired. Only low resolution spectra are necessary, thanks to rapid (thousands of $\mathrm{km} \mathrm{s}^{-1}$ ) expansion velocities in SNe. Since nearby supernovae usually have their peak output in the optical, but have important lines distributed from the blue to the red (Filippenko 1997), an ideal spectrograph would be low resolution, have high efficiency, and cover the entire optical range. There is much to learn from time-resolved spectra of supernovae, but even classification spectra benefit from the ability to respond quickly to new discoveries.

9. RV vetting of transiting planet candidates: The dimming of starlight due to planetary transits can be mimicked by grazing binary stars. However, such transits also induce reflex motions in their parent star as they orbit a common center of mass, which can be detected by monitoring the star's radial velocity (Mayor \& Queloz 1995). The reflex signals of planets are at most a few hundred $\mathrm{m} \mathrm{s}^{-1}$, while those of binary stars are typically many $\mathrm{km} \mathrm{s}^{-1}$. Thus, accuracy better than about a hundred $\mathrm{ms}^{-1}$ is necessary, requiring a medium-to-high resolution spectrograph $(R \geq 40,000)$. The target stars are often bright, obviating the need for a large aperture telescope, but multiple observations, distributed in time, are needed to detect orbital motion. In the near future, NASA's TESS mission (Ricker et al. 2010) will provide many thousands of candidate planets circling bright stars, all requiring such observations. Higher-precision radial velocities (a few $\mathrm{m} \mathrm{s}^{-1}$ ) allow one to determine masses of transiting planets down to Neptune's mass or smaller.

10. The future: We doubt that all of the interesting applications of time-domain observation have yet been explored, in part precisely because there are, as yet, no suitable observing facilities to support them. We hope that, going forward, the LCOGT network will inspire astronomers to find applications that are not yet envisioned.

\subsection{Education and Public Engagement Activities}

From its inception, LCOGT has been committed to using astronomy as a tool for public engagement and informal education, with the aim of encouraging technically-minded learners of all ages to think critically and develop investigative skills. We have, however, found it surprisingly difficult to translate this commitment into effective action in the context of the public schools. The obstacle is that the resource that LCOGT can offer in relative abundance is telescope time, and not (as would be more traditional for a nonprofit education organization) support for teacher training, nor for curriculum development.

In consequence, we concentrate our educational efforts in two areas in which we can be effective. First, LCOGT provides access to observing time on its telescopes, to schools, to astronomy clubs and similar organizations, and sometimes to individuals. Some of this time is used in the form of real-time observing, in which users operate telescopes remotely. Recently, however, we have provided a larger portion as queue-scheduled observing, just as for science observations.

Second, we have invested significant effort in the development of online tools and activities for education and public engagement. LCOGT develops programs for citizen scientists, people who, irrespective of their training or experience, can become knowledgeable enough that they are able to assist in (and get recognition for) obtaining and analyzing observations that may be used to carry out genuine scientific investigations. These tools are necessarily web-based, and hence they have the potential to reach people with a diverse range of abilities and backgrounds, and in large numbers. Some of these tools complement telescope use, whereas others provide a rich web-based experience for a general audience, as is discussed more in $\S 8$.

\section{NETWORK STRUCTURE-OVERVIEW}

What features must a telescope network have to meet the observing challenges suggested above? This question was prominent in our thinking from LCOGT's beginning; to help us address it, we have relied heavily on discussions with the community, especially as embodied in our advisory structure. This structure consists of a board of trustees, an additional small group of counselors who serve with no definite term, and a much larger rotating-membership scientific advisory committee. With the help of these advisors, we defined both the broad science goals to be pursued and the key features that a network must have in order to achieve the goals. We address several of these features below, notably the number and geographical distribution of nodes, the number and size of telescopes, the instruments that are attached to those telescopes, and the software for coordinating network observations and for making reduced data available.

Our description of these features is complicated by the incompleteness of the LCOGT Network at the time of this writing and uncertainties regarding future funding, and therefore the 
speed of construction and ultimate extent of the Network's facilities. For clarity's sake, we thus describe the Network and its evolution in terms of 3 completion phases, which we label A, B, and C. Phase A consists of all of the Network features that are operating as of the date of this writing (2013 April). Phase B consists of additional facilities that LCOGT has funding to deploy, and that we expect to complete before roughly 2014 January. The union of features in Phases A and B is thus a reasonable expectation for the minimum capabilities of the completed Network. These phases have been financed mostly by contributions from the TABASGO Foundation (our primary donor), with generous additional funding from the Scottish University Physics Alliance (SUPA), administered through St. Andrews University. Phase C consists of facilities beyond Phase B, which we would like to deploy as soon as possible, but on a schedule that will be driven by the availability of funding beyond that committed by the TABASGO Foundation. Phase C is essentially our vision of the "final" Network configuration. Since we do not plan to close down any facilities in the process of Network development, Phase $\mathrm{C}$ facilities include all of Phases A and B, and Phase B includes all of Phase A.

If the funding sought for Phase $\mathrm{C}$ should fail to materialize, the damage to the Network's scientific capabilities would be mostly a quantitative degradation in number and quality of observations, rather than a qualitative change in the kinds of science that could be done. Fewer telescopes would mean fewer observations could be carried out per year, and would also limit scheduling flexibility. Probably the largest effects would be noticed in the northern hemisphere, where Phase A and B plans call for few sites and telescopes. Hence, continuous observations would not be possible north of roughly $30^{\circ}$ declination, and a few programs that require concurrent observations could easily saturate the small number of available $1 \mathrm{~m}$ telescopes.

\subsection{Number and Geographical Distribution of Nodes}

A minimum requirement for a network to be fully capable of time-domain operation is that it should allow continuous time coverage of objects located anywhere in the sky. To achieve this requires at least three well-separated nodes in each of the northern and southern hemispheres. Thus, from three ideally spaced mid-latitude sites, each separated from the others by eight hours of time, night-times overlap by about four hours in the hemisphere's winter, and hardly at all in the summer. Also, northernhemisphere sites cannot see the southern circumpolar stars, and conversely. Therefore, six sites is the minimum feasible for true global coverage.

When choosing potential node sites, we first aimed to keep cost manageable by considering only sites that are already developed and are occupied by functioning astronomical facilities. This not only avoids significant infrastructure development costs, but also facilitates access to trained staff when needed. Beyond this requirement, the science we wish to do defines our desiderata. In order of decreasing importance, these are: many good observing hours per year, good seeing, dark sky, good prospective scientific collaborations, and good internet access.

In practice, this simple and deterministic picture is complicated by geography (e.g., the northern hemisphere has a wider choice of acceptable astronomical sites than the southern), by the physical and bureaucratic accessibility of the sites involved, and by the nature of existing commitments, collaborations, and facilities (e.g., LCOGT had operating telescopes in Australia and Hawaii before we began to plan the $1 \mathrm{~m}$ network). With these considerations in mind, we settled on a Phase $\mathrm{C}$ configuration of seven main nodes and one secondary node, three in the southern hemisphere and five in the northern. ${ }^{8}$ In the southern hemisphere, these are Cerro Tololo Inter-American Observatory in Chile (CTIO), Siding Spring Observatory in Australia (SSO), and the South African Astronomical Observatory at Sutherland, South Africa (SAAO). The northern (Phase C) main nodes are McDonald Observatory in Texas (MDO), the Haleakala Observatory on the island of Maui (HO), the Xinjiang Astronomical Observatory near Urumqi, China (XAO), and the Teide Observatory on the island of Tenerife, in the Canary Islands (TO). There are four sites in the north both because of the longitudinal distribution of suitable sites, and because, though we have a $2 \mathrm{~m}$ telescope in Hawaii, we have not yet succeeded in obtaining permits to place $1 \mathrm{~m}$ telescopes there. The secondary node is the Byrne Observatory at Sedgwick (BOS) in California near our headquarters; although we do not expect to make this node a full part of the Network, it is useful for instrument testing, software development, and ad hoc longitude-specific backup of Network observations.

Table 1 lists the characteristics of these nodes, including the completion status (Phases denoted PA, PB, or PC) of the telescopes intended for each. To summarize the table, we have six Phase-A nodes (CTIO, SSO, SAAO, MDO, HO, BOS). These support 10 Phase-A telescopes: $2 \times 2 \mathrm{~m}$ telescopes at SSO and $\mathrm{HO}, 7 \times 1 \mathrm{~m}$ telescopes at CTIO, SAAO, and MDO, and the $83 \mathrm{~cm}$ telescope at BOS. Phase B will add one additional $1 \mathrm{~m}$ telescope to MDO, and $2 \times 1 \mathrm{~m}$ telescopes to SSO. We anticipate that a total of $11 \times 40 \mathrm{~cm}$ telescopes will be added at these sites through 2014 as part of Phase B (see Table 1). If completely funded, Phase $\mathrm{C}$ will add $2 \times 1 \mathrm{~m}$ telescopes at $\mathrm{HO}$, and two additional nodes (TO and $\mathrm{XAO}$ ) including $3 \times 1 \mathrm{~m}$ telescopes at TO, $2 \times 1 \mathrm{~m}$ telescopes at XAO, and about 12 additional $40 \mathrm{~cm}$ telescopes distributed among various nodes.

\subsection{Number and Size of Telescopes}

Choosing the size of telescope for the Network, and the number of telescopes per node, involved tradeoffs among the per-telescope cost, the per-telescope performance, and network system considerations. A key point proved to be that network

\footnotetext{
${ }^{8}$ Please see http://lcogt.net/network.
} 
TABLE 1

LCOGT NeTwork Node CHARACTERISTICS

\begin{tabular}{|c|c|c|c|c|c|c|c|c|c|}
\hline Node & Latitude (degree) & E. Long (degree) & Elev (m) & Seeing $(")$ & Sky bright $\left(\mathrm{mag}^{\prime \prime-2}\right)$ & $2 \mathrm{~m}$ & $1 \mathrm{~m}$ & $83 \mathrm{~cm}$ & $40 \mathrm{~cm}$ \\
\hline CTIO ..... & -30.1673 & -70.8046 & 2180 & 0.9 & 22.0 & - & (PA:3) & - & (PB:3 \\
\hline MDO $\ldots .$. & +30.6800 & -104.0151 & 2070 & 1.3 & 22.1 & - & (PA:1,PB:2) & - & (PC:3 \\
\hline BOS $\ldots \ldots$ & +34.6915 & -120.0422 & 360 & 2.5 & 20.7 & - & - & (PA:1) & - \\
\hline $\mathrm{HO} \ldots \ldots . .$. & +20.7070 & -156.2575 & 3040 & 1.2 & 22.0 & (PA:1) & (PC:2) & - & (PB:2) \\
\hline SSO ........ & -31.2729 & 149.0708 & 1160 & 1.4 & 21.5 & (PA:1) & $(\mathrm{PB}: 2)$ & - & (PB:2) \\
\hline XAO $\ldots \ldots$ & +43.4723 & 87.1760 & 2080 & - & 21.7 & - & (PC:2) & - & (PB:2) \\
\hline SAAO $\ldots . .$. & -32.3806 & 20.8101 & 1780 & 1.3 & 22.1 & - & (PA:3) & - & (PB:2) \\
\hline TO ........ & +28.3004 & -16.5115 & 2390 & - & - & - & (PC:3) & - & (PC:3 \\
\hline
\end{tabular}

NOTES.-Column descriptions: (1) node name, (2) north latitude (degrees), (3) east longitude (degrees), (4) elevation (m), (5) median seeing full width at half maximum ("), (6) typical sky brightness (equivalent $V$ magnitude per square "), (7) number of 2 m telescopes, with development phase (PA = Phase A, etc.), (8) as column 7, but number of $1 \mathrm{~m}$ telescopes, (9) as column 7, but number of $83 \mathrm{~cm}$ telescopes, (10) as column 7, but number of $40 \mathrm{~cm}$ telescopes. An entry of "--" means "none" or "inadequate information".

scheduling flexibility is greatly improved by having at least two telescopes at each node. Moreover, since we expect spectroscopy to be an important part of our observation mix, and since spectroscopic observations tend to involve long integration times (and hence are awkward to interrupt), we planned to put 3 telescopes at as many sites as we could afford. Last, holding collecting area equal, it is less costly to build many small telescopes than one large one. One of our earliest activities was thus to design and build a number of $40-\mathrm{cm}$ telescopes. This was both to validate design concepts to be applied to larger instruments, and eventually to offload some educational and other bright-object observations from our $2 \mathrm{~m}$ telescopes to smaller, more cost-effective ones. On the other hand, more telescopes means more components to fail, and the relative cost of instruments grows as telescope apertures shrink. And, of course, a certain minimum aperture is required to do the kinds of science for which the network is intended.

After considering all this (and more), we concluded that the best compromise was to build $1 \mathrm{~m}$ telescopes, with the intention of placing three telescopes at each node. Recent economic events have made this goal temporarily unreachable, but as a long-term goal we continue to aim for a Phase $\mathrm{C}$ network having 2 or $3 \times 1 \mathrm{~m}$ telescopes per node at each of seven nodes, for a total of $17 \times 1 \mathrm{~m}$ telescopes.

\section{TELESCOPES}

Our goal is to provide a homogeneous network of robotic telescopes, with identical instrumentation, which will allow continuous monitoring of astronomical targets from both hemispheres.

We currently operate (Phase A) or plan to operate (Phase B, C) the following facilities:

1. The $2 \mathrm{~m}$ Faulkes Telescopes (FTN at Haleakala, Hawaii, and FTS at Siding Spring, Australia) (Phase A)

2. The $1 \mathrm{~m}$ Telescope Network, with nodes at sites worldwide (Phase A, B, C)

3. The $83 \mathrm{~cm}$ telescope, at the Byrne Observatory at Sedgwick in California (Phase A)

4. The $40 \mathrm{~cm}$ Telescope Network, also at sites worldwide (Phase B, C)

Optical and mechanical characteristics of each of these telescope classes are given in Table 2; we describe each telescope class in more detail in the remainder of this section.

\subsection{Faulkes 2 m Telescopes}

The two Faulkes Telescopes (Fig. 1) were designed and constructed by Telescope Technologies Limited (TTL). The

TABLE 2

LCOGT Telescope Characteristics

\begin{tabular}{|c|c|c|c|c|c|c|c|c|}
\hline Aperture & $\mathrm{f} / \#$ & Mount & Corr. FOV (') & Img. scale " pix ${ }^{-1}$ & Slew rate $\left(\right.$ degrees $\mathrm{s}^{-1}$ ) & Instruments & AGs & \# Avail. (PC) \\
\hline $2 \mathrm{~m}$ & $\mathrm{f} / 10$ & Alt/Az & 15 & 0.155 & 2 & 5 & 4 & 2 \\
\hline $1 \mathrm{~m}$ & $\mathrm{f} / 8$ & Equatorial & 46 & 0.386 & 6 & 5 & 1 & 17 \\
\hline $83 \mathrm{~cm} \ldots .$. & $\mathrm{f} / 8$ & Equatorial & 15 & 0.483 & 7 & 3 & 2 & 1 \\
\hline $40 \mathrm{~cm} \mathrm{\ldots ...}$ & $\mathrm{f} / 8$ & Equatorial & 36 & 0.965 & 10 & 2 & 1 & 23 \\
\hline
\end{tabular}

NOTES. - Column descriptions: (1) telescope aperture, (2) Cassegrain focus f/ratio, (3) type of telescope mounting, (4) corrected field of view diameter (arcmin), (5) Cassegrain image scale (arcsec per $15 \mu \mathrm{m}$ pixel), (6) slewing speed (degrees per second), (7) number of instrument ports (excluding dedicated autoguiders), (8) number of available system autoguiders, (9) number of telescopes of this type available for deployment (Phase C). 


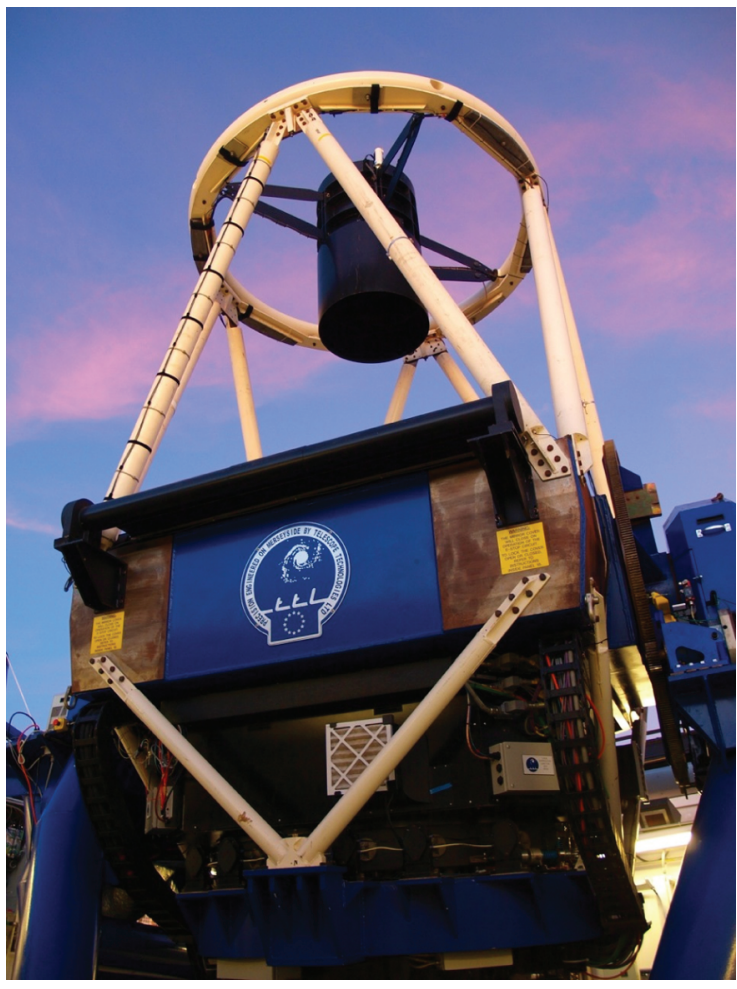

FIG. 1.-LCOGT's Faulkes North (FTN) $2 \mathrm{~m}$ telescope at dusk, with the clamshell enclosure open. Faulkes South (FTS) is a twin of FTN, but located at Siding Spring Observatory in Australia.

telescopes feature Ritchey-Chrétien Cassegrain f/10 optics, with solid primary mirrors of Astro-Sital, $20 \mathrm{~cm}$ thick, and secondaries of the same material, on Alt-Az mounts. We correct minor astigmatism in the current secondary mirrors using flexure assemblies driven by compressed-air pistons. These astigmatic secondaries will soon be replaced with lightweighted AstroSital mirrors. Primary mirror supports consist of 36 air-pressure driven support pads, which are automatically adjusted to compensate for elevation-dependent flexure. The secondary mirrors are adjustable in focus. Secondary mirror positions are adjusted dynamically (but only when the instrument shutters are closed) to compensate for changes in telescope elevation angle and in ambient temperature.

The $2 \mathrm{~m}$ mountings are Alt-Az designs, with natural frequencies in excess of $10 \mathrm{~Hz}$. Oil-pad bearings support both altitude and azimuth motions; A large ball-bearing-supported Cassegrain rotator compensates image rotation. We drive all axes with opposed servo motors and gear boxes, with feedback provided by motor encoders and by optical tape encoders attached to the driven parts. The maximum slewing speed in all axes is 2 degrees/s, and the servo settling time is less than $5 \mathrm{~s}$. Thus, pointing to new objects seldom takes longer than $45 \mathrm{~s}$. Blind pointing is accurate from 3 to $10^{\prime \prime}$ depending on the Cassegrain axis position. Periodic tracking errors due to the

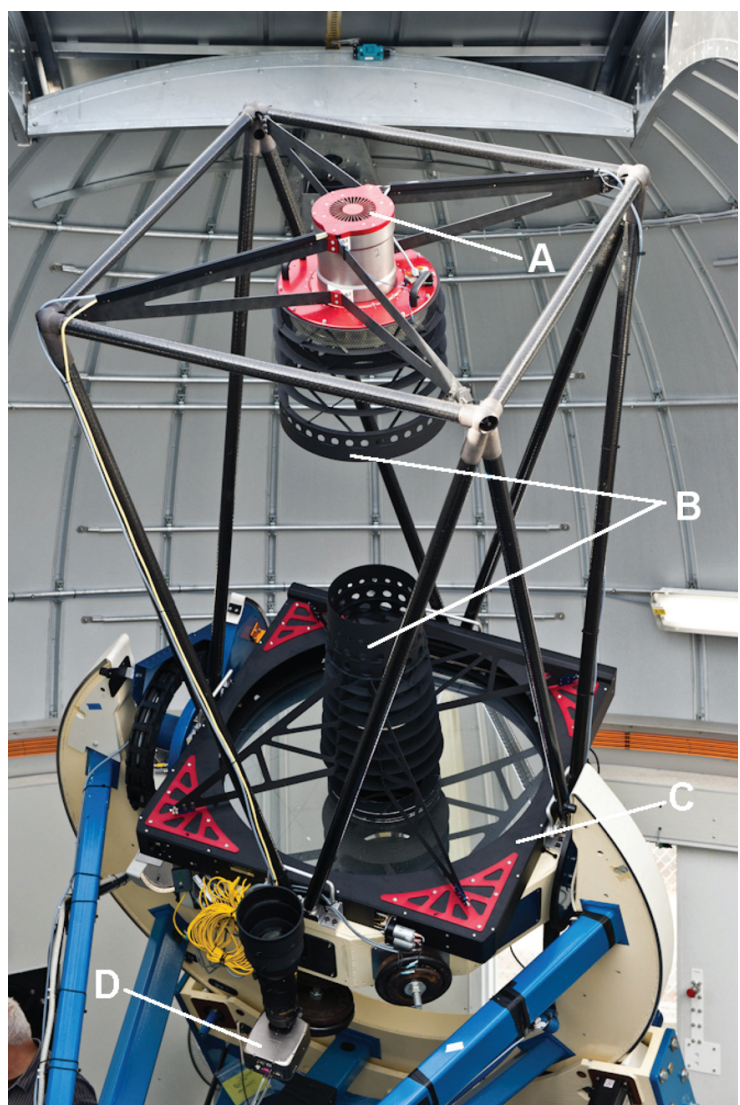

FIG. 2.-1 m telescope, assembled in dome. (A) Secondary mirror tip-tilt-focus mechanism. (B) Lightweight, low-wind-resistance light baffles. $(C)$ Roller-shade primary mirror cover, with integral Hartmann mask. $(D)$ Wide-field Extinction Camera. Photo: Matt Miller, HazardousTaste.com.

influence of gear teeth are minimized using correction lookup tables.

Up to five different instruments can be mounted at the Cassegrain focus, one in the straight through position and four smaller ones on side ports accessible by rotating the science fold tertiary mirror. Imaging instruments available on the telescopes are the "Spectral" (Fairchild Imaging CCD486) and "Merope" (E2V-4240) optical cameras, and a lucky imaging and highspeed imaging (LIHSP) camera. We recently deployed on each of the Faulkes telescopes a low-resolution cross-dispersed spectrograph (known as FLOYDS) with $320 \mathrm{~nm}$ to $1100 \mathrm{~nm}$ coverage at better than $1.2 \mathrm{~nm}$ spectral resolution. All of these instruments are described in more detail in $\S 5$.

The $2 \mathrm{~m}$ telescopes are housed in large (10 m square) clamshell enclosures, with shutters operated by hydraulic pistons. When open, the clamshells allow unvignetted views of the sky for all elevations greater than $20^{\circ}$ above the horizon. In use, these enclosures have proved to be reliable and leak-free. They also provide space to deploy additional $40 \mathrm{~cm}$ telescopes on elevated platforms within the $2 \mathrm{~m}$ enclosure. 


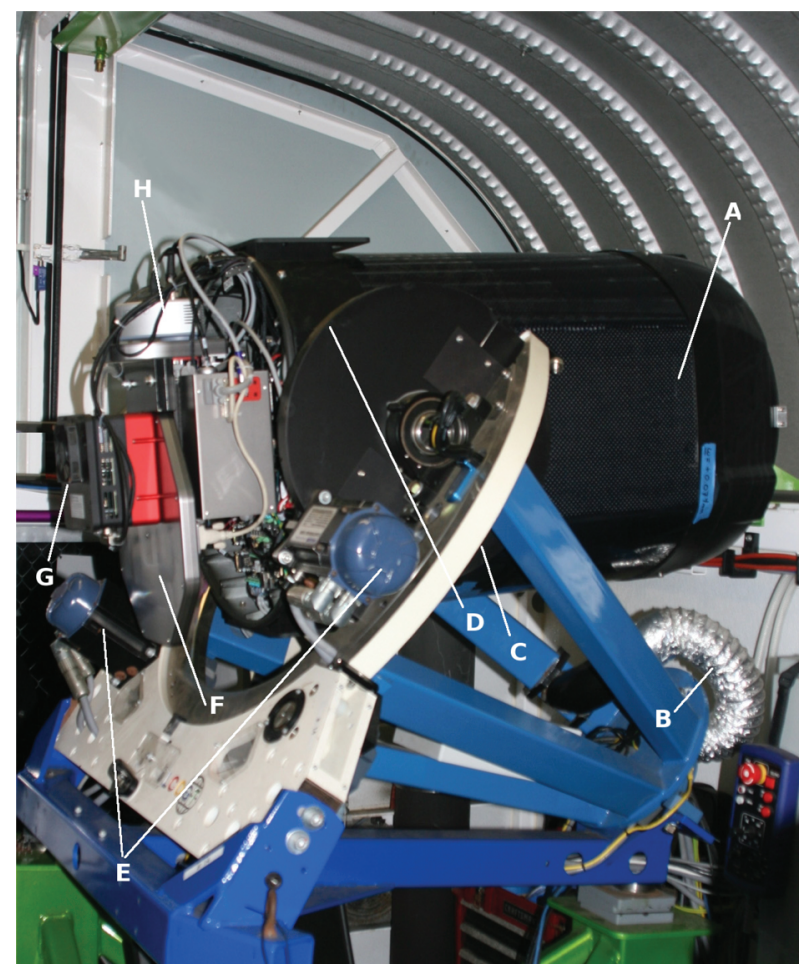

FIG. 3. $-40 \mathrm{~cm}$ telescope in its Aqawan enclosure. (A) Carbon-epoxy telescope tube. $(B)$ Duct for optics tube ventilation. (C) Polar axis drive ring, with white fabric dirt guard. $(D)$ Declination axis drive ring. $(E)$ Direct-drive servo motors for R.A. (left) and decl. (right) axes. (F) 8-position filter wheel for main science camera. $(G)$ SBIG STX-6303 main science CCD camera. $(H)$ Andor Luca R LIHSP EMCCD camera.

\subsection{The 2 m Robotic Control System}

The two Faulkes Telescopes are controlled by a Robotic Control System (RCS) (Fraser \& Steele 2004). The RCS runs on a local computer at the site in parallel to the Telescope Control System (TCS), which controls the telescope functions such as slewing, tracking, autoguiding, etc., and the Instrument Control System (ICS), which controls the functions of the instruments. While the telescope is operating, it is the RCS that issues instructions to the TCS and ICS. The system features built-in recovery functions to address problems automatically, such as an instrument failing to initialize properly (Mottram 2006). Additionally, the telescopes have local weather stations which serve information on humidity levels, cloud cover, precipitation, wind speed, and temperature to the RCS. If any of these parameters should exceed their allowed ranges the enclosure is automatically closed and the system goes into standby mode.

When atmospheric conditions (seeing, extinction) are too poor to allow normal science observations, the RCS switches to observing background standard stars. These are observed fairly frequently in order to assess changes in atmospheric conditions so that normal operation can resume when the atmospheric parameters return to within their acceptable limits.

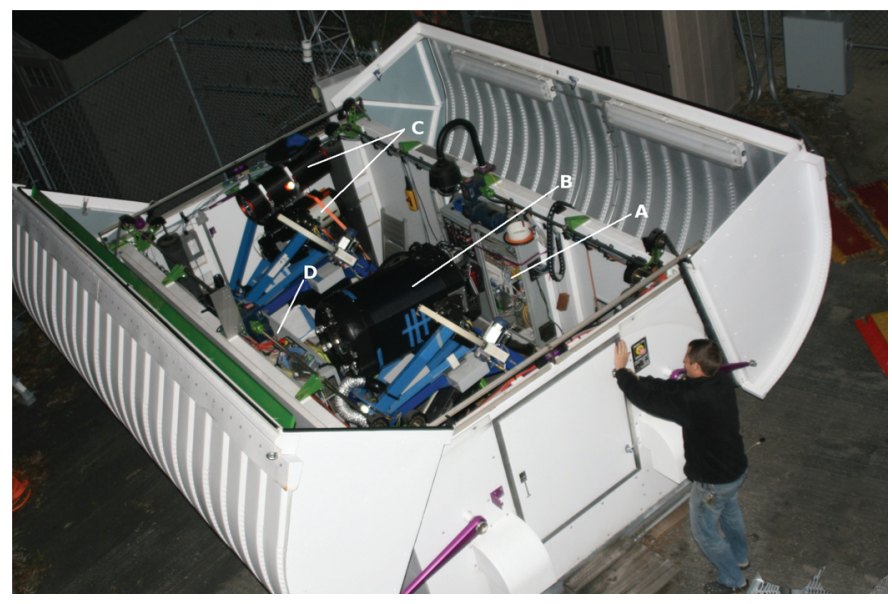

FIG. 4.-Aqawan enclosure in the fully open position. (A) Electronics control system for Aqawan enclosure. (B) $40 \mathrm{~cm}$ telescope in C-ring mount. (C) $20 \mathrm{~cm}$ astrograph (top) and spectrograph for measuring atmospheric extinction (bottom) in a single C-ring mount. $(D)$ Electronics control system for two C-ring mounts.

For science observations, the RCS receives instructions from a database of observations, known as the Phase-II DB. This is stored locally at each telescope site and contains all the observing programs with their specifications. The RCS uses this information to determine the set of instructions to issue to the telescope and instrument systems. The actual scheduling and submission of the observing requests to the telescopes is handled by a separate "dispatch" scheduler. This runs in real-time; whenever an observation is completed, it lists the observations in the Phase II DB that are then able to run, ranks these in order of priority (which is a complex function of many variables, some of which depend on current observing circumstances), and dispatches the highest-ranked feasible observation to be executed by the telescope.

The RCS accepts input from two sources. The first one is the Observer Support System (OSS). This controls access to the database of observations uploaded by the users to the telescope or submitted automatically via external agents. The second source of input is the Target of Opportunity Control System (TOCS). This is an override program whose execution interrupts the observing schedule and initiates immediate observations of a high priority target. The TOCS can be started automatically via external triggers or may be invoked manually. Typical examples of frequent users of this system are responses to alerts of GammaRay bursts and anomalous microlensing events. The OSS can respond to astronomical alerts within minutes (Saunders et al. 2008), whereas requests through the TOCS can put a telescope on a new target and begin observing within tens of seconds.

We are in the process of replacing the software just described with a structure that is compatable with that for the $1 \mathrm{~m}$ Network (described below). This replacement will occur in phases, with the aim of completing the transition by the end of 2014. This replacement will maintain almost all of the capabilities of the 
old system, while adding valuable new features, notably the ability to schedule observations that involve coordination between the $2 \mathrm{~m}$ telescopes and the $1 \mathrm{~m}$ Network.

\subsection{Archiving}

The image archiving system for the $2 \mathrm{~m}$ telescopes is separate from and predates the $1 \mathrm{~m}$ system, which is described in $\S$ 6.6. In the $2 \mathrm{~m}$ system, incoming images are transferred from each telescope to the central archives every 10 minutes. They are then available on a quick-look page so that, if required, an initial assessment of the data quality can be made. We calibrate the images hosted on the quick-look pages using the most recent flat-fields and biases, usually from the previous night. However, since new twilight flats are obtained automatically at the end of each night, we recalibrate the images using the latest flat-field frames before storing them in the permanent archive. Data are filed under the proposal name that generated them, with password-protected access for a proprietary period (normally one year, for scientific data). Data taken for educational purposes are made available to the public immediately.

\section{4. $1 \mathrm{~m}$ Telescopes}

The $1 \mathrm{~m}$ telescopes in the global Network (Fig. 2) are designed to fulfill LCOGT's objective to provide homogeneous, maximally available optical monitoring of time-variable sources. Each $1 \mathrm{~m}$ telescope must provide reliable robotic operation for long periods of time, with minimal hands-on maintenance; deliver good pointing, tracking, and guiding; and provide uniform image quality. We manufactured optical and mechanical parts to tight tolerances to achieve uniform image scale and consistent performance among telescopes and instruments.

The reasons for choosing a C-ring equatorial mount are detailed in Dubberley (2010) but summarized here for completeness. Alt-Az mounts offer obvious mechanical advantages for larger telescopes, but dramatically increase the dynamic range requirements for the drive servo. As well, an Alt-Az configuration would require the addition of a Cassegrain rotator axis under servo control, resulting in undesirable complexity and weight for the $1 \mathrm{~m}$ telescope class. The $\mathrm{C}$-ring concept has long been known to provide accurate pointing and tracking performance (cf. Palomar $5 \mathrm{~m}, \mathrm{NOAO}$ and AAT $4 \mathrm{~m}$ ), and we were also influenced by the known long-term robotic reliability of the 2MASS telescopes. C-rings are not popular for visual astronomy because of the difficulty of human eye access to their focus, or for telescopes requiring frequent facility instrument changes, but they provide a stable and reliable mount, with sufficient instrument clearance for our purposes. Total instrument depth below the bolt-circle on the back of the primary mirror cell is $650 \mathrm{~mm}$, with an overall Optical Tube Assembly (OTA) length (including instrument) of $2.5 \mathrm{~m}$.

The LCOGT $1 \mathrm{~m}$ telescopes are modular, each comprising 15 basic structural, optical, and control components, which we ship to site in a few prealigned assemblies. Completed mounts weighing two tons (base, $2 \mathrm{~m}$-diameter C-ring and mirror-cell) are aligned in our shop, shipped to site, and craned through the $2.1 \mathrm{~m}$ open shutter aperture of our domes onto their metal pedestals and concrete piers, with the primary mirror about $2 \mathrm{~m}$ above ground level. Each telescope is compact, fitting inside a $6 \mathrm{~m}$ diameter modified Ash-dome. Minimum clearance within the dome is only about $100 \mathrm{~mm}$ in a few locations. The telescopes can point and track under the pole, and within $2^{\circ}$ of the pole, but the C-ring limits east-west tracking to just over $5 \mathrm{hr}$ from the meridian, conforming to our specified horizon limit of $15^{\circ}$ or 3.7 airmasses.

The OTA design and finite element analysis details are given in Haldeman et al. (2010) and again summarized here. We considered a traditional Serrurier truss design, but a central box section would have required a larger C-ring and heavier OTA and mount. Instead, we adopted a "Gemini"-like concept in which the Mirror cell forms both the declination axis and the main load bearing structure for the OTA. The mirror cells are weldments that were each post-machined in one pass for precise alignment of the instrument bolt circle, and of the optical support points. The C-ring opening is pinched slightly during assembly and held together by the mirror cell; these contrasting forces preload the mount, reducing movement between parts as loads change during telescope motion.

We designed the optical system, described in Haldeman et al. (2010), for good image quality, usually limited by site seeing, and for photometry over a large enough field to provide many stars for astrometric and relative flux measurements. Our final $1 \mathrm{~m}$ optical design comprises an $\mathrm{f} / 2.5$ Hextek lightweight primary and $330 \mathrm{~mm}$ diameter Hextek secondary, both optically finished by LZOS in Russia, providing an f/8 modified RitcheyChrétien system with the addition of a doublet corrector in front of the instrument package. The system is designed for $80 \%$ enclosed energy within a circle of diameter $0.6^{\prime \prime}$. We paid considerable attention to optimizing the baffling against stray light from astronomical sources, such as the moon, to allow for good air flow, and to minimize ghosting in the Cassegrain field.

Our lightweight Hextek borosilicate mirrors have an expansion coefficient of $3.3 \mathrm{ppm} \mathrm{C}^{-1}$ at typical operating temperatures. We employ a traditional 18-point whiffle tree design for the primary mirror axial support, and constrain the mirror in the transverse directions using a stainless steel hub attached to the upper surface of the instrument bolt circle, with two slightly springy plastic rings to press against the inner diameters of the top and bottom primary glass plates. This results in measured primary movements of order $70 \mu \mathrm{m}$ radially and axially during telescope motion from the zenith to horizon. But these motions are repeatable, and hence can be compensated by appropriate terms in the telescope mount model. We find that hysteresis in these motions is $10 \mu \mathrm{m}$ or less (about $4^{\prime \prime}$ projected on the sky). To hold the secondaries without inducing thermal stresses, we fabricated powder cast and post-machined central 
hubs of invar tuned to the same CTE as the glass, and epoxied into the secondary mirror central holes prior to polishing. We then support the secondary mirror hubs by a compact 3-axis system that provides focus and remote tilt collimation to submicron repeatability. We set mirror alignment transverse to the optical axis during telescope assembly.

Both primary and secondary mirrors move slightly and change their radii as a result of changing ambient temperature and telescope zenith angle, which in turn cause variations in telescope focus, collimation, and pointing. We largely compensate collimation errors by tuning the support system so that there is substantial cancellation of the primary and secondary mirror tilts with zenith angle. We compensate the remaining collimation errors, as well as errors in focus, by adjusting the secondary mirror in tip, tilt, and position along the optical axis. Focus adjustments take place when they exceed half of the optically measurable limit, and automatically occur between science exposures, when the main instrument shutter is closed. We absorb residual errors in pointing into the telescope mount model.

$1 \mathrm{~m}$ telescope pointing is currently about 6" RMS over the sky. Good polar alignment is critical to avoid differential image motion between the science imager and off-axis guiders. This is being achieved to a few arcsececonds by a combination of analysis with Tpoint software, and flash World Coordinate System (WCS) fitting via astrometry.net. The $1 \mathrm{~m}$ telescopes currently slew at $6 \mathrm{deg} \mathrm{s}^{-1}$ (slower when the safety system detects the presence of people in the dome), and the dome azimuth drives have been upgraded so that the telescope can move to tracking on any new source in $30 \mathrm{~s}$ or less. Open loop (unguided) tracking error is about $2 \mathrm{arcsec} / \mathrm{hr}$. Guided tracking is accurate to better than $0.5^{\prime \prime}$ or about one pixel.

Our Hextek primaries contain 108 air "pockets", each about 1-liter in volume. These are open at the bottom, and tend to trap warm air as the night cools. This leads to slight "dimpling" of the surface, visible in pupil images, and also to significant focus and spherical aberration concerns due to non-thermalization of the primary. The secondary is largely immune to this effect as its holes face up and warm air escapes, but we do provide a fan system drawing air across M2. We were able to overcome these disturbing effects in M1 by routing a "sucker" system with a plastic tube into each of the 108 pockets, continuously sucking the air out to be replaced by air at ambient temperature.

We cool cryogenic detectors with closed-cycle refrigerators (Cryotiger), and warmer instruments and electronics with a circulating refrigerated propylene glycol mixture. We vent all removed heat directly to the outside of the telescope enclosure. We maintain the air surrounding the primary and OTA at ambient temperature by a combination of dome fans drawing outside air in through the open slit, and mirror cell fans drawing air down over the primary and out the back of the cell. The combination of fans and sucker system maintains excellent thermalization of the primary, removes dimpling, and minimizes residual spherical aberration.

\subsection{Byrne Observatory at Sedgwick Telescope}

The Byrne Observatory at Sedgwick Reserve (BOS) hosts a $0.83 \mathrm{~m}$ telescope built around an RC Optical Systems ${ }^{9}$ optical tube assembly. The observatory was developed in agreement with UC Santa Barbara in the hills near Santa Ynez, CA. The land is part of the University of California natural reserve system. BOS is located $60 \mathrm{~km}$ from LCOGT headquarters, in much darker skies. It is nevertheless only about $25 \mathrm{~km}$ from the Pacific ocean, so it frequently suffers from high humidity and fog, especially during the summer months. The facility is used nightly by LCOGT staff and UCSB students for robotic observations, and also hosts about a dozen star parties per year.

The classical Ritchey-Chrétien optical system has a primary mirror diameter of $83 \mathrm{~cm}$, and a secondary of $29.8 \mathrm{~cm}$. Working at $\mathrm{f} / 7.97$, the focal length is $6.615 \mathrm{~m}$, giving a plate scale of $31.5 \mu \mathrm{m} \mathrm{arcsec}^{-1}$ at the focal plane. The equatorial fork style mount was designed and built by LCOGT in 2007-2008. Along with its $6 \mathrm{~m}$ modified Ash-dome, it was intended as a full scale prototype to prove the friction drive, motors, and enclosure now used on the $1 \mathrm{~m}$ telescopes. We chose a fork mount over a C-ring for the BOS site to allow eyepiece access for public outreach. The mount can reach $\left(\mathrm{HA} \pm 5.3 \mathrm{hr}\right.$, elevation $>20^{\circ}$ ), and slews at a maximum rate of 7.5 degrees $^{-1}$. The telescope, instruments, and dome are routinely run under remote or robotic control.

For two years BOS ran independently of the nascent $1 \mathrm{~m}$ Network, and proved effective as a tool for science, for public outreach, and for astronomy course support at UCSB. It has now been upgraded to use the same mechanisms and software systems as the broader Network. In this form it will add a new role as testbed for the NRES spectrograph prototype (see $\S 5.6 .2$ ).

\section{6. $40 \mathrm{~cm}$ Telescopes}

LCOGT's $40 \mathrm{~cm}$ class of telescopes (Fig. 3) is based on a Meade 16-inch telescope, but with extensive modifications to all moving parts. We have assembled 23 of these telescopes, though in Phase A, none of these have been permanently deployed to remote sites. Phase B calls for deploying 11 of them to five nodes, as shown in Table 1.

Our modifications of the telescopes consist of rework on the OTA to ensure stable focus and collimation and to provide thermal control, and also construction of a custom equatorial mount to carry the OTA. This mount is a C-ring design that shares the hardware drive and software control mechanisms of our $1 \mathrm{~m}$ mounts. Indeed, we used the $40 \mathrm{~cm}$ mount development as an opportunity to prototype the $1 \mathrm{~m}$ mount, and it proved very useful in clarifying and providing solutions for problems inherent in this basic design.

\footnotetext{
${ }^{9}$ Please see http://www.rcopticalsystems.com/.
} 
The $40 \mathrm{~cm}$ optical specifications are given in Table 2. In sky tests of several of these optical systems from our offices near Santa Barbara, we have found that the telescopes deliver sub-arcsecond imaging when they are in thermal equilibrium and accurately collimated. To keep the telescopes close to the ambient temperature, we use a ducted fan to circulate filtered air from outside the enclosure through the inside of the OTA.

We will mount most of the Phase-B $40 \mathrm{~cm}$ telescopes on steel piers roughly $1 \mathrm{~m}$ high, within small clamshell enclosures that we have dubbed Aqawans (Fig. 4). ("Aqawan" is a Chumash word meaning "to keep dry".) Each Aqawan has room for two telescopes, placed so that they can move independently to any location on the sky, with the enclosure roof open or closed (or partly open), without the telescopes colliding with the enclosure structure or with each other. The mountings slew at up to 10 degrees $^{-1}$, settle and begin tracking within $5 \mathrm{~s}$, and with software mount modeling, achieve blind pointing accuracy of about $10^{\prime \prime}$ RMS for targets anywhere above the telescopes' $15^{\circ}$ elevation limit. The C-ring mount design allows sky access anywhere up to $\pm 4.7 \mathrm{hr}$ from the meridian. Pointing and tracking below the celestial pole is allowed.

The LCOGT-designed Aqawan enclosures are inspired by but substantially modified from enclosures built for the MONET telescopes (Bischoff et al. 2006). They are $4.3 \mathrm{~m}$ long by $2.8 \mathrm{~m}$ wide, and $2.6 \mathrm{~m}$ tall at the roof peak. Their two roof segments have potential to injure people as they open or close, so we install Aqawans only in fenced and locked areas, and we provide multiple emergency stop buttons both inside and outside the enclosure. Control of the enclosures is provided through a custom-designed electrical panel built around an industrial Programmable Automation Controller. We provide battery backup for the electrical power needed to open and (especially) to close the Aqawans, and should this fail, one may open or close them manually. We have tested the enclosures through thousands of open-close cycles, and they display great reliability. But unfortunately (for this purpose) our Santa Barbara test facility experiences a limited range of extreme weather. We therefore anticipate with interest the arrival of the first Aqawan at a site where snow, ice, and high winds are regular occurrences.

\subsection{Site Environmental Monitoring System (SEMS)}

The core requirements of our environmental system at each site are to monitor temperature, humidity, dewpoint, wind speed and direction, wetness, particulates, barometric pressure, and sky brightness (solar insolation in daytime, sky brightness in $\mathrm{V}$ mag $\operatorname{arcsec}^{-2}$ at night). Temperatures and humidities are monitored for each site, and at several places for each telescope, so critical functions like enclosures and mirror covers can be closed when necessary. We extend our monitoring to incorporate data from other tenants' site weather instruments as well as our own. In some cases these instruments add information not measured by the SEMS instrument suite, and even redundant data are useful, since they provide frequent sanity checks for the SEMS data. Additionally we provide clean dry air outlets to our $1 \mathrm{~m}$ mirrors that can be activated to prevent dewing.

Our typical limits are that telescope enclosures can be open when the humidity is less than $90 \%$, the dewpoint(s) are more than $2 \mathrm{C}$ below ambient or any mirror temperatures, the windspeed is less than $18 \mathrm{~m} \mathrm{~s}^{-1}\left(65 \mathrm{~km} \mathrm{hr}^{-1}\right)$, particulate count at $1.0 \mu \mathrm{m}$ is less than $10^{6} \mathrm{~m}^{-3}$, and solar zenith distance is greater than $85^{\circ}$. The latter condition allows us to open and thermalize before sunset, stay open after sunrise, and to capture twilight flat fields, typically obtained at a point $105^{\circ}$ away from the Sun, which tends to minimize the spatial gradients in light from the sky (Chromey \& Hasselbacher 1996).

We employ a Campbell Scientific CR1000 data logger, with Vaisala pressure and temperature/humidity sensors, Boltwood cloud sensors, Unihedron SQM-LE sky brightness monitors, and other sensors. These are mounted on a 6-m tall tower, typically attached to the side of our Site Services Building. At sites such as McDonald Observatory that can be affected by lightning, we also deploy an electric field sensor. The SEMS system is powered through the site Uninterruptible Power Supply (UPS) to carry it through short power outages. If power remains off for longer periods, or if the SEMS heartbeat is not detected, our safety system starts a sequenced shutdown to close all enclosures.

In addition to hard environmental constraints we have adopted softer constraints such as those based on transparency estimates. We estimate sky transparency via calibrated Boltwood (sky minus ambient) temperature sensors and, when we are open, we can also estimate transparency directly via our science and context cameras, based on measured magnitudes of known catalog stars.

SEMS data are sampled every $12 \mathrm{~s}$, and persist for seven days. They are then resampled on a timescale of $120 \mathrm{~s}$, which persists in our database for 2 years. They are then resampled once more on a timescale of $1200 \mathrm{~s}$, which persists indefinitely. In this way we can match current or historical data or telescope events to the conditions prevailing at the time.

Additionally, we are developing a comprehensive Network Telescope Operations browser interface, for both internal and external use. This provides an overview of our Network, summarizes the status of each site and telescope, and provides graphical monitoring of the environmental systems described above. $^{10}$

\subsection{Safety Systems}

We have implemented a comprehensive set of safety systems into our global Network. These include interlocks on enclosure doors and gates, panel doors and ladders (stowed or not) that could interfere with telescope motion. We have a Fortress Interlocks trap-key system, within which keys to open enclosures or

\footnotetext{
${ }^{10}$ Please see https://telops.lcogt.net.
} 
gates must first be removed from a monitored access panel in the Site Services Building (SSB). This informs the system of the presence and location of people. Typically the presence of people does not prevent tracking of affected telescope(s), but does limit their slew-speed. If enclosure lights are left on, we automatically extinguish them when the enclosure opens.

In addition to access control, all telescopes and enclosures have carefully designed systems for the safety of personnel and equipment. Each enclosure and telescope can be in one of three states: automatic (for full robotic control), manual for local control only, and disabled for maintenance. Enclosures cannot open and telescopes cannot move if deliberately left in manual or disabled mode. Our telescopes each have four levels of limit switches: L1 software limit, L2 electrical sensor position limit (also used for homing each axis), L3 electrical shutoff, and L4 hardstop. Automatic recovery is possible only from the first two of these. Both $2 \mathrm{~m}$ and $1 \mathrm{~m}$ telescopes have failsafe air brake systems; caliper brakes on both axes are kept off by power and air pressure, and come on if either power or pressure fails. The brakes are also turned on if an error condition occurs.

Our servo systems provide continuous checks for overspeed or out-of-bounds operation. Our Programmable Automation Controllers (PACs) provide checks on safe operation for all subsystems. Additionally on our $1 \mathrm{~m}$ Network we have implemented a "PNOZ" configurable safety system from Pilz Automation, programmed to detect and react to a variety of situations. A severe condition may result in an emergency stop (E-stop) being activated, which stops and prevents all further motion on that telescope and enclosure; on-site human interaction is required to investigate, troubleshoot, and recover from such a situation. If danger presents, humans can also press red E-stop buttons distributed around each enclosure and telescope.

Less critical abnormal conditions may result in one or more components being de-activated, or reset, according to the programmed reaction. Complex moving interactions such as upper and lower shutters of our domes are programmed into this safety system: The upper shutter must open before the lower shutter can start, and the upper shutter may pause before full closure to first allow the lower shutter to close completely.

\subsection{Thermal Management}

The Site Services Building contains automatic fans and a large air conditioning unit to maintain temperature of the building and all computers near to a $20^{\circ} \mathrm{C}$ set point. The NRES bench spectrograph (see $\S 5.6$ ) will be placed within its own container adjacent to the SSB, and will incorporate even more stable thermal control than that within the SSB.

Electrical panels within our enclosures are normally ventilated outside the enclosure with fans. These fans can be automatically turned off, or pulsed in cold, wet conditions, to prevent influx of damp air which could cause internal dewing. All telescope enclosures are painted white to reduce heat-load during daytime. Each dome contains a small air conditioning unit that comes on automatically in hot weather to reduce internal temperatures and minimize thermalization issues when the domes open. Each dome has three large wall fans that come on at a controllable speed when the enclosures open. These draw ambient air through the open shutter and out of the enclosure, and completely replace the air in each dome within a few minutes. Thermalization of the whole telescope environment to ambient temperature typically occurs within 30-60 minutes, depending on temperature differentials between day and nighttime at the site. As of 2013 February we have operated in nighttime temperatures in the range from $-7^{\circ} \mathrm{C}$ (McDonald in the winter) to $+30^{\circ} \mathrm{C}$ (Chile in the summer), but typically not changing by more than $10^{\circ} \mathrm{C}$ on any given night at any site. We have designed for an operating range from $-15^{\circ} \mathrm{C}$ to $+40^{\circ} \mathrm{C}$.

Each enclosure contains a circulating glycol cooling system, maintained at a temperature exceeding the local dewpoint, used to circulate heat away from thermoelectrically cooled cameras, guiders, and electronics crates. The heat from this system is exhausted outside each enclosure with fans. $1 \mathrm{~m}$ mirror cells contain eight fans that draw air over the mirrors and out the back of each cell. The sucker system fan comes on when each dome opens to thermalize each $1 \mathrm{~m}$ primary, again typically within about 30 minutes.

The LCOGT-designed Sinistro camera (covered in detail in the next section) is cooled to $-100^{\circ} \mathrm{C}$ with recirculating gas from a Cryotiger unit in a cryo-cabinet inside each dome. The hoses for this system and the glycol system run through the RA and DEC energy chains to the primary mirror cell.

\section{INSTRUMENTS}

LCOGT's instrument complement is designed to support a range of science goals in time-domain astrophysics. Our aim is that every telescope offer a standardized suite of instruments kept as homogeneous as possible for each class of telescope, across all nodes in the network. This enables observations to be carried out promptly by the best available node, or for time series observations of a given target to be carried out by a sequence of telescopes around the globe, as night falls at each node.

LCOGT instrumentation falls into two categories: imagers and spectrographs. Our software control system allows any imager to be used as an autoguider, including self autoguiding. Instruments designed specifically for autoguiding (these include facility autoguiders dubbed " $4 \mathrm{ag}$ " and " $2 \mathrm{m0} 0$ " that are mounted on the $2 \mathrm{~m}$ telescopes, and the " $1 \mathrm{~m} 0$ " autoguider for the $1 \mathrm{~m}$ telescopes) have independent focus control. For bright-star photometry, this allows in-focus images on the autoguider and simultaneous defocused images on the science camera. By design, the autoguider mounts minimize flexure relative to the primary instrument's image plane, and have been verified to be stiff against gravity deflection to about 0.1 resolution element. 
Table 3 lists the imaging instruments that are installed on the various LCOGT telescopes. Although homogeneity among imagers is highly desirable, this desire is often outweighed by the need to accommodate differing image scales among telescopes, differing readout requirements and fields of view among main science cameras, high-speed cameras, and autoguiders, and the necessity to deploy telescopes promptly, even though the imagers ultimately intended for them may not yet be ready. For these

TABLE 3

LCOGT NeTWORK IMAGER CHARACTERISTICS

\begin{tabular}{|c|c|c|c|c|c|}
\hline $\begin{array}{l}\text { Instrument name } \\
\text { Camera type } \\
\text { Detector type } \\
\end{array}$ & $\begin{array}{l}\text { Detector format } \\
\text { Plate scale }\end{array}$ & $\mathrm{QE}_{\max }$ & $\begin{array}{l}\text { Readout } \\
\quad(\mathrm{s})\end{array}$ & $m(1 e)\left(r^{\prime}\right)$ & Filters \\
\hline \multicolumn{6}{|l|}{ Merope (2.0) } \\
\hline $\begin{array}{l}\text { Merope } \ldots \ldots \ldots \ldots \ldots \ldots \\
\text { e2v CCD42-40 DD, BI } \ldots \ldots \ldots \\
\text { Spectral }(2.0) \ldots \ldots \ldots \ldots \ldots \ldots\end{array}$ & 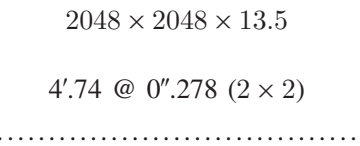 & $90 \%$ & 14 & 24.6 & $\begin{array}{l}u^{\prime} g^{\prime} r^{\prime} i^{\prime} z_{s} Y B V R_{C} I_{J} \mathrm{H} \alpha \mathrm{H} \beta[\mathrm{O} \mathrm{III}] \mathrm{DDO} 51 \\
V+R \mathrm{ND} 2 V_{s}\end{array}$ \\
\hline $\begin{array}{l}\text { Spectral } 600 \quad \ldots \ldots \ldots \ldots \ldots \\
\text { FI CCD486 BI } \quad \ldots \ldots \ldots \ldots \ldots \\
\text { LIHSP }(2.0) \quad \ldots \ldots \ldots \ldots \ldots \ldots \ldots\end{array}$ & 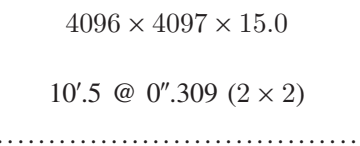 & $90 \%$ & 11 & 24.6 & $\begin{array}{l}u^{\prime} g^{\prime} r^{\prime} i^{\prime} z_{s} Y U V B V R_{C} I_{C} \mathrm{H} \alpha \mathrm{H} \beta[\mathrm{O} \text { III] DDO51 } \\
V+R \mathrm{ND} 2 V_{s}\end{array}$ \\
\hline $\begin{array}{l}\text { Andor iXon } 888 \ldots \ldots \ldots \ldots \\
\text { e2v CCD201 BI, FT, EM } \ldots \ldots \\
\text { SBIG }(1.0) \quad \ldots \ldots \ldots \ldots \ldots \ldots \ldots\end{array}$ & 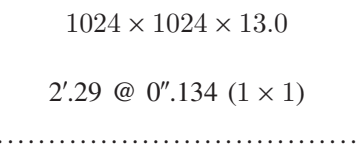 & $90 \%$ & 0.13 & 23.9 est. & $\begin{array}{l}u^{\prime} g^{\prime} r^{\prime} i^{\prime} z_{s} Y B V R_{C} I_{J} \mathrm{H} \alpha \mathrm{H} \beta[\mathrm{O} \mathrm{III}] \mathrm{DDO} 51 \\
V+R \mathrm{ND} 2 V_{s}\end{array}$ \\
\hline $\begin{array}{l}\text { SBIG STX-16803 } \ldots \ldots \ldots \ldots \\
\text { Kodak KAF-16803 FI } \quad \ldots \ldots \ldots \\
\text { Sinistro }(1.0) \quad \ldots \ldots \ldots \ldots \ldots \ldots\end{array}$ & $\begin{array}{c}4096 \times 4096 \times 9.0 \\
15^{\prime} .8 @ 0^{\prime \prime} .464(2 \times 2) \\
\ldots \ldots \ldots \ldots \ldots \ldots \ldots \ldots \ldots\end{array}$ & $50 \%$ & 12 & 23.0 & $u^{\prime} g^{\prime} r^{\prime} i^{\prime} z_{s} Y w U V B u V R_{C} I_{C}$ \\
\hline $\begin{array}{l}\text { Sinistro (LCOGT) } \quad \ldots \ldots \ldots \ldots \\
\text { FI CCD486 BI } \quad \ldots \ldots \ldots \ldots \ldots \\
\text { Autoguider }(1.0) \quad \ldots \ldots \ldots \ldots \ldots\end{array}$ & $\begin{array}{c}4096 \times 4097 \times 15.0 \\
26^{\prime} .4 @ 0^{\prime \prime} .387(1 \times 1) \\
\ldots \ldots \ldots \ldots \ldots \ldots \ldots \ldots \ldots\end{array}$ & $90 \%$ & 4 & 23.5 est. & $u^{\prime} g^{\prime} r^{\prime} i^{\prime} z_{s} Y w U V B u V R_{C} I_{C}$ \\
\hline 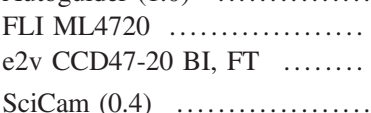 & $\begin{array}{c}1024 \times 1024 \times 13.0 \\
5^{\prime} .72 @ 0^{\prime \prime} .335(1 \times 1)\end{array}$ & $90 \%$ & 1 & 23.8 & $g^{\prime} r^{\prime} i^{\prime} z_{s} B u V$ Clear \\
\hline $\begin{array}{l}\text { SBIG STX-6303 } \ldots \ldots \ldots \ldots \ldots \\
\text { Kodak KAF-6303E FI } \ldots \ldots \ldots \\
\text { SBIG }(0.8) \quad \ldots \ldots \ldots \ldots \ldots \ldots\end{array}$ & $\begin{aligned} 3072 \times 2048 \times 9.0 \\
29^{\prime} .7 \times 19^{\prime} .8 @ 0^{\prime \prime} .580(1 \times 1)\end{aligned}$ & $50 \%$ & 12 & 22.1 & $u^{\prime} g^{\prime} r^{\prime} i^{\prime} z_{s} w B u V$ \\
\hline $\begin{array}{l}\text { SBIG STL-6303E } \ldots \ldots \ldots \ldots \\
\text { Kodak KAF-6303E FI } \quad \ldots \ldots \ldots \\
\text { Merope }(0.8) \quad \ldots \ldots \ldots \ldots \ldots \ldots\end{array}$ & $\begin{array}{c}3072 \times 2048 \times 9.0 \\
14^{\prime} .8 \times 9^{\prime} .90 @ 0^{\prime \prime} .580(2 \times 2)\end{array}$ & $50 \%$ & 20 & 23.2 & $B V g^{\prime} r^{\prime} i^{\prime} z^{\prime} \mathrm{H} \alpha$ Clear \\
\hline $\begin{array}{l}\text { Merope } \ldots \ldots \ldots \ldots \ldots \\
\text { e2v CCD42-40 DD, BI } \ldots \ldots \ldots \\
\text { LIHSP }(0.8) \ldots \ldots \ldots \ldots \ldots \ldots\end{array}$ & $\begin{array}{c}2048 \times 2048 \times 13.5 \\
14^{\prime} .8 @ 0^{\prime \prime} .43513 .5(1 \times 1)\end{array}$ & $90 \%$ & 14 & 23.8 est. & $\begin{array}{l}u^{\prime} g^{\prime} r^{\prime} i^{\prime} z_{s} Y B V R_{C} I_{J} \mathrm{H} \alpha \mathrm{H} \beta[\mathrm{O} \mathrm{IIII}] \text { DDO51 } \\
V+R \text { ND2 } V_{s}\end{array}$ \\
\hline $\begin{array}{l}\text { Andor Luca R } \ldots \ldots \ldots \ldots \ldots \\
\text { TI TX285SPD-B0 BI, EM } \ldots \ldots \\
\text { LIHSP }(0.4) \quad \ldots \ldots \ldots \ldots \ldots \ldots\end{array}$ & $\begin{array}{c}1004 \times 1002 \times 8.0 \\
4^{\prime} .29 @ 0^{\prime \prime} .257(1 \times 1)\end{array}$ & $50 \%$ & 0.13 & 21.7 est. & $g^{\prime} r^{\prime} i^{\prime} z_{s} B u V$ Clear \\
\hline $\begin{array}{l}\text { Andor Luca } R \quad \ldots \ldots \ldots \ldots \ldots \\
\text { TI TX285SPD-B0 BI, EM } \ldots \ldots \\
\text { LIHSP }(1.0) \quad \ldots \ldots \ldots \ldots \ldots \ldots\end{array}$ & $\begin{array}{c}1004 \times 1002 \times 8.0 \\
8^{\prime} .60 @ 0^{\prime \prime} .515(1 \times 1)\end{array}$ & $50 \%$ & 0.13 & 20.1 est. & $g^{\prime} r^{\prime} i^{\prime} z_{s} B u V$ Clear \\
\hline $\begin{array}{l}\text { Andor iXon } 888 \ldots \ldots \ldots \ldots . \\
\text { e2v CCD201 BI, FT, EM } \ldots \ldots \\
\text { ExtCam }(1.0) \quad \ldots \ldots \ldots \ldots \ldots \ldots\end{array}$ & $\begin{array}{c}1024 \times 1024 \times 13.0 \\
5^{\prime} .72 @ 0^{\prime \prime} .335(1 \times 1)\end{array}$ & $90 \%$ & 0.13 & 22.1 est. & $g^{\prime} r^{\prime} i^{\prime} z_{s} B u V$ Clear \\
\hline $\begin{array}{l}\text { SBIG STL-6303E } \quad \ldots \ldots \ldots \ldots \\
\text { Kodak KAF-6303E FI } \quad \ldots \ldots \ldots\end{array}$ & $\begin{array}{c}3072 \times 2048 \times 9.0 \\
238^{\prime} \times 158^{\prime} @ 4^{\prime \prime} .64(1 \times 1)\end{array}$ & $50 \%$ & 10 & 17.7 est. & $B u V r^{\prime} i^{\prime} z_{s}$ \\
\hline
\end{tabular}

NOTES.-Column descriptions: (1) instrument name, (telescope aperture in m), generic type of dewar/readout electronics, manufacturer's designation of detector chip, (2) detector format shown as (X-dimension) $\times($ Y-dimension $) \times$ (pixel size in $\mu \mathrm{m})$, plate scale shows field of view in arcmin, projected pixel size in arcsec at the indicated binning (e.g., $2 \times 2$ ), (3) maximum detector quantum efficiency (percent), (4) full image readout time at the binning shown in column 2, (5) stellar magnitude in $r^{\prime}$ producing 1 photoelectron per s, (6) list of filters normally mounted on the imager. 
reasons, the Network employs a considerable variety of CCD imagers. In the remainder of this section, we describe these instruments in more detail.

\subsection{Sinistro}

Sinistro is the standard multi-instrument package which will be mounted on all $1 \mathrm{~m}$ telescopes (Fig. 5). Corrector optics offer a straight-through unobstructed and unvignetted $116 \mathrm{~mm}$ diameter field to the primary science imager. As an interim measure for the $1 \mathrm{~m}$ telescopes we have shipped to date, the CCD camera attached to this imager is an SBIG STX-16803, a frontside illuminated $4 \mathrm{~K} \times 4 \mathrm{~K}$ device with $9 \mu \mathrm{m}$ pixels giving a field of view $15^{\prime} .8$ arcmin square (see Table 3 ). This camera uses a Peltier cooler and operates at $-20^{\circ} \mathrm{C}$.

The interim Sinistro CCD camera will soon (Phase B) be replaced with a Fairchild Imaging CCD 486 BI $4096 \times 4096$ device with $15 \mu \mathrm{m}$ pixels. This is run by an LCOGT designed and built detector controller, which achieves a $4 \mathrm{M} \mathrm{pixs}^{-1}$ readout with a read noise of $\sim 10 e^{-}$pix $^{-1}$. The image scale is $25 . " 8 \mathrm{~mm}^{-1}$ or $0 .{ }^{\prime \prime} 387 \mathrm{pix}^{-1}$, giving a field of view 26.6 square. The instrument is maintained at $-100^{\circ} \mathrm{C}$ in an LCOGT-designed cyrostat, cooled by a Brooks Automation PCC Cryotiger. Sinistro's LCOGTdesigned filter system comprises three independent overlapping wheels, each holding seven square, $75 \mathrm{~mm}$ filters, $3-8 \mathrm{~mm}$ thick. This system can change filters within $5 \mathrm{~s}$ and provides 21 usable filter slots. We list the filters available in LCOGT imagers in Table 4; in addition to the filters shown there, Sinistro wheels each have a pinhole for optical testing and two spare slots. The $1 \mathrm{~m}$

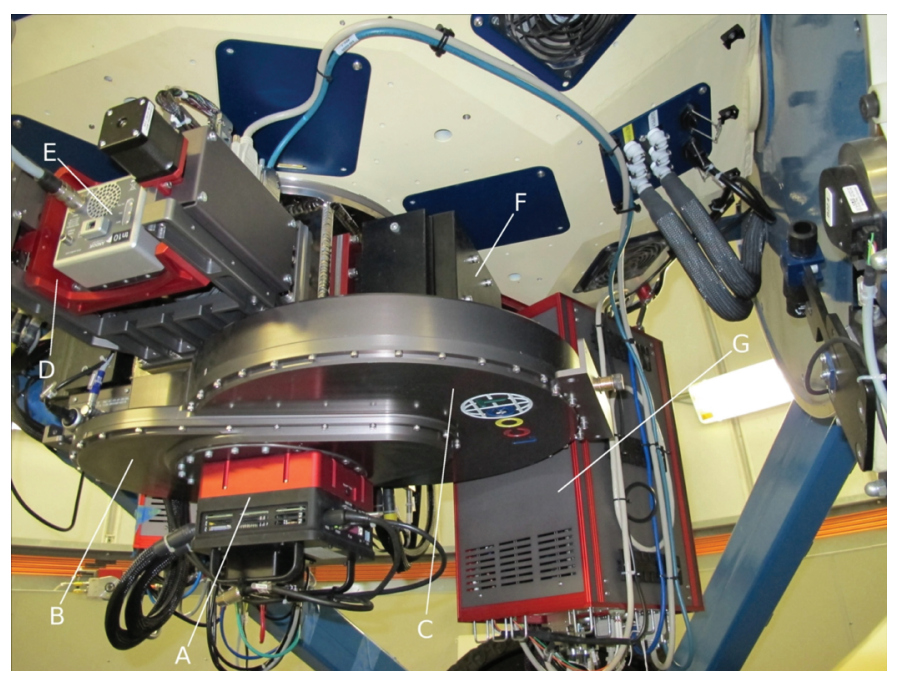

FIG. 5.-Sinistro instrument package mounted on the tailpiece of a $1 \mathrm{~m}$ telescope. (A) SBIG STX-16803 $4 \mathrm{~K} \times 4 \mathrm{~K}$ CCD camera. $(B)$ Rotating-disk shutter assembly. (C) 3-layer filter wheel assembly. $(D)$ Side port with independent focus stage for LIHSP camera or autoguider. $(E)$ Andor Luca S LIHSP camera; may also be used as autoguider. ( $F$ ) Side port (one of 4 ) with blank-off panel. $(G)$ Electronics crate for tailpiece power distribution, signal conditioning, and related functions telescope focus automatically adjusts as required by filter changes, with the focus offsets specified in configuration files, but most filters are in fact parfocal.

Sinistro also offers four off-axis ports that, in the absence of seeing, would produce diffraction-limited images. The first supports a dedicated, independently focusable autoguider based on a high-QE back-illuminated frame-transfer CCD (e2V 4720). The second houses an independently focusable high speed camera described in $\S 5.4$. The third is connected to the fiber feed to the NRES spectrograph described in $\S 5.6$, while the fourth port is open for future instruments. These side ports use off-axis regions of the $1 \mathrm{~m}$ telescope's field, so that deployable turning mirrors are not required. Since all instruments view the sky all the time, it is in principle possible to use multiple instruments simultaneously; this capability is currently implemented only for guiding during science exposures.

\subsection{BOS Imaging Instruments}

BOS is outfitted for traditional CCD imaging, as well as high speed photometry/lucky imaging. The telescope's primary science camera is a Santa Barbara Instrument Group ( $\mathrm{SBIG}^{11}$ ) STL-6303E with a $3072 \times 2048$ Kodak KAF-6303E CCD, TEC+glycol chilled to $-20^{\circ} \mathrm{C}$, and mounted to an SBIG filter wheel equipped with filters as listed in Table 4. We will soon (Phase B) replace this camera with a copy of the Merope instrument used on the $2 \mathrm{~m}$ telescopes, with properties listed in Table 3. The BOS primary science camera is supported by two unfiltered off-axis autoguiders with independent focus stages to allow for intentionally defocused observations.

An Andor Technology ${ }^{12}$ Luca $R$ camera is used for high speed photometry, lucky imaging, and speckle interferometry. The $1004 \times 1002 \times 8 \mu \mathrm{m}$ EMCCD can image at $12.4 \mathrm{~Hz}$ (full frame). The focal length is boosted with a $2 \times$ Barlow lens for a pixel scale of $0^{\prime \prime} .1286$ pixel $^{-1}$ and a $2^{\prime} .1 \times 2^{\prime} .1$ field of view. This camera can provide diffraction-limited imaging in the optical even under poor seeing conditions, and high-speed photometry of bright sources. The Andor camera can quickly and easily be removed and replaced with an eyepiece for visual observing during star parties. Due to its close proximity to LCOGT's headquarters near Santa Barbara, CA, BOS is a testbed for prototype instruments and now hosts the prototype of LCOGT's NRES echelle spectrograph.

\subsection{Filter Selection}

Our aim is to employ a range of filters spanning the near-UV $(\sim 320 \mathrm{~nm})$ to near-IR $(\sim 1000 \mathrm{~nm})$ which exploit the wavelength-sensitivity of our detectors to achieve our science goals. We chose broadband filters from two photometric systems: Landolt (Johnson/Cousins) and SDSS (Sloan primed) owing

\footnotetext{
${ }^{11}$ Please see http://www.sbig.com/.

${ }^{12}$ Please see http://www.andor.com/.
} 
1044 BROWN ET AL.

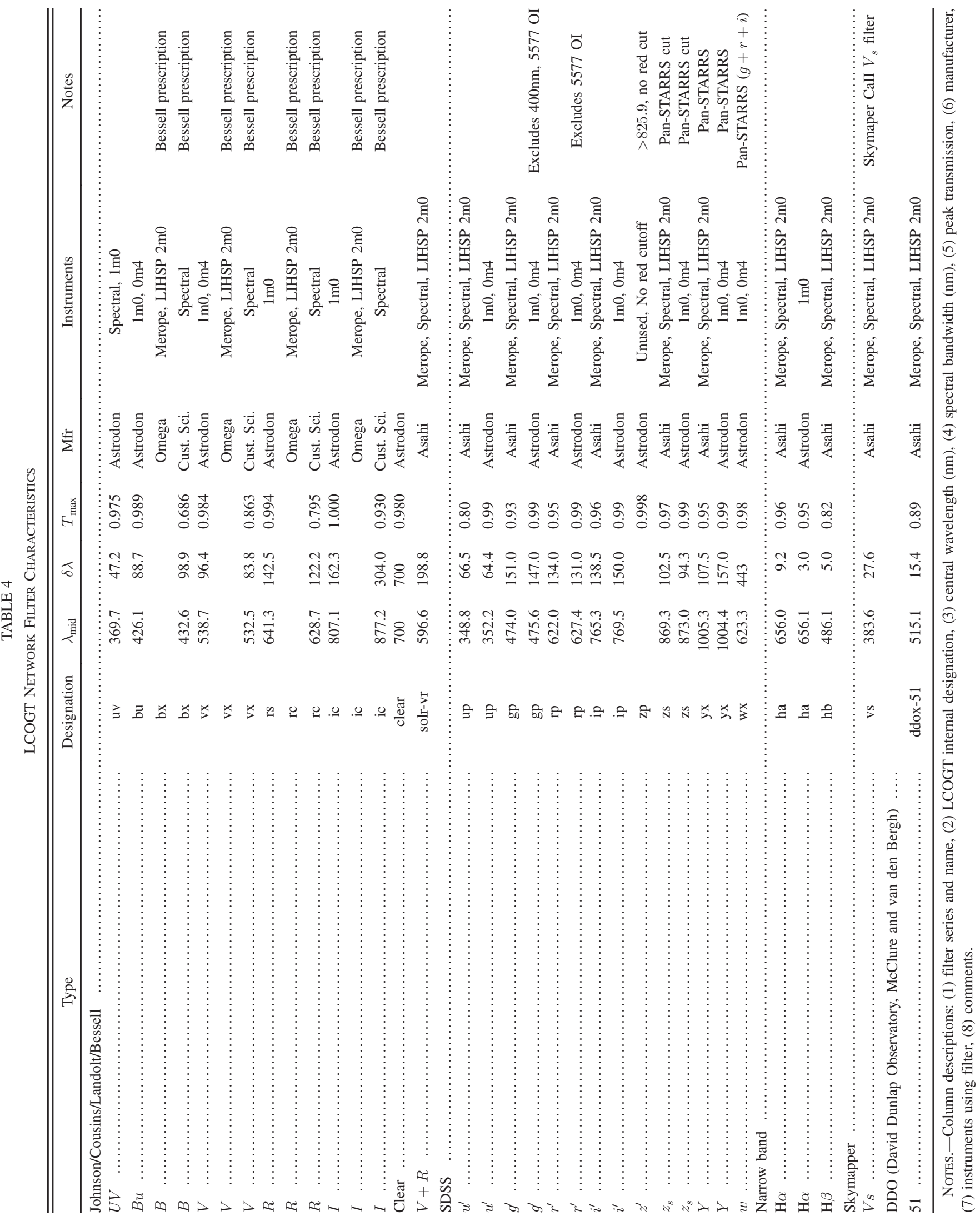


to their widespread use in astronomy. After careful consideration of the glass, coatings and exact prescriptions used by various manufacturers, we selected Astrodon filters as providing the closest match to the original filter response functions of each photometric standard. On delivery of each filter, we measure the true transmission curve and store it in a database. To use the strong red sensitivity of our CCDs we will also have broadband Pan-STARRS- $Z_{S}$ and $-Y$ filters. For science requiring high throughput, such as asteroid imaging, we will also have the Pan-STARRS-w filter. Table 4 lists the names and characteristics of filters that may be found on LCOGT instruments. The LCOGT webpage contains links to the filter transmission curves, CCD quantum efficiency versus wavelength, and measured mirror reflectance versus wavelength.

\subsection{Lucky Imaging and High-Speed Photometers}

Several of LCOGT's scientific goals require occasional highresolution imaging. Examples include photometry of microlensed stars in very dense starfields in the galactic bulge, searches for background eclipsing binaries in the close neighborhood of suspected transiting-planet candidates, and detection and characterization of binary asteroids. Moreover, occultation studies of solar system objects and eclipses of compact stars demand high temporal sampling rates. Unfortunately, present adaptive-optics sytems remain too expensive for us to deploy to all of our sites. We have therefore chosen to install Electron Multiplying CCD (EMCCD) detectors on both of the Faulkes Telescopes and on four of our $1 \mathrm{~m}$ telescopes (one at each of four sites). These enable high speed imaging for both high speed photometry and resolution enhancement techniques (e.g., "lucky imaging"). We refer to these instruments generically as LIHSP: lucky imaging and high speed photometers.

High cadence imaging permits photometry of events that require short time-typically subsecond-monitoring (e.g., occultations [Elliot 1979]). Moreover, the ability to collect rapid measurements of astronomical targets allows a variety of resolution enhancing techniques: lucky imaging (Law et al. 2006; Garrel et al. 2012), speckle interferometry (Lohmann et al. 1983; Stelzer \& Ruder 2007), and image deconvolution (Hirsch et al. 2011) all require short exposure $(>10 \mathrm{~Hz}$, on bright targets, typically $R<14$ for the $2 \mathrm{~m}$ and $R<12$ for our $1 \mathrm{~m}$ telescopes) to achieve spatial resolution beyond the limitations imposed by the atmospheric effects of image smearing.

All of the techniques mentioned above are implemented at LCOGT, where we have achieved a spatial resolution of $0.3^{\prime \prime}$ at the Faulkes Telescopes. Although lucky imaging techniques generally should allow diffraction limited imaging, the $2 \mathrm{~m}$ optical systems are not diffraction limited. Although Barlow lenses decreasing the plate scale may achieve better than $0.3^{\prime \prime}$ resolution, we do not expect to be able to reach the diffraction limit on the $2 \mathrm{~m}$ telescopes. Results may be better for our smalleraperture systems; we expect heavy use of the $40 \mathrm{~cm}$ telescopes to produce high-resolution images for education and outreach.
For the $2 \mathrm{~m}$ and $1 \mathrm{~m}$ telescopes, the high speed imaging camera is an Andor iXon 888 using a back-illuminated EMCCD, while many of the $40 \mathrm{~cm}$ telescopes will host an Andor iXon Luca R with a front-illuminated EMCCD.

In EMCCDs, a solid state electron multiplying (EM) register added to the end of the normal serial register amplifies the signal before the readout amplifier introduces noise. Thus EMCCDs allow the detection of signal in photon starved conditions. A particularly useful application in astronomy is high cadence imaging. The cameras installed on our $2 \mathrm{~m}$ and $1 \mathrm{~m}$ telescopes allow imaging at a cadence as high as $8 \mathrm{~Hz}$ on the full field: $2^{\prime} .3$ square at the FTs, and $5^{\prime} .7$ square at the $1 \mathrm{~m}$ telescopes, with a pixel resolution of $0^{\prime \prime} .13 \mathrm{pix}^{-1}$ and $0 . " 34 \mathrm{pix}^{-1}$ respectively. On the $40 \mathrm{~cm}$ telescopes the Luca $\mathrm{R}$ allows full field imaging at $12.4 \mathrm{~Hz}$ cadence, with a field of view measuring $4^{\prime} .3$ square, and a plate scale of $0 . " 26 \mathrm{pix}^{-1}$. The final image scale and field of view is still to be determined for all telescopes based on science needs, and may be modified with the use of Barlow lenses. Experience suggests that on the $2 \mathrm{~m}$ Faulkes Telescopes, a scale of $0^{\prime \prime} .07 \mathrm{pix}^{-1}$ may be better. Subframing and binning allow us to achieve higher cadences: tens or even hundreds of Hz. We typically operate the detectors in frame transfer mode, so the duty cycle for each exposure cycle is $>99 \%$ at $8 \mathrm{~Hz}$, and still $>50 \%$ at the highest cadences available.

\subsection{FLOYDS}

The FLOYDS instruments are a pair of nearly identical, low dispersion, robotic spectrographs deployed at the $2 \mathrm{~m}$ Faulkes Telescopes, North and South. The instruments were designed with supernova classification and monitoring in mind, with a very large wavelength coverage ( $\sim 320$ to $1000 \mathrm{~nm})$ and a resolution ( $R \sim 300$ to 600 , depending on wavelength) wellmatched to the broad features, but heterogenous nature, of these transient events. The FLOYDS spectrographs are also excellent for other monitoring programs (e.g., reverberation mapping of active galactic nuclei) where the robotic nature of the spectrographs allow for campaigns not previously possible with classically scheduled spectrographs. A more detailed discussion of the FLOYDS instruments, our design choices, and first science will be presented in a forthcoming work (Sand et al., in preparation).

The chosen design uses a low dispersion grating $\left(2351 \mathrm{~mm}^{-1}\right)$ and a cross-dispersed prism in concert to work in first and second order simultaneously. A folded all-reflecting camera focuses first- and second-order light onto the CCD. This allows a $\sim 320$ to $1000+\mathrm{nm}$ wavelength coverage in two orders in a single exposure. A $30^{\prime \prime}$ slit length allows both orders to fit on the chip with no order overlap (Fig. 6, top). Four such 30" length slits are available at each spectrograph, with widths that bracket the median seeing at each site, and a 6 " slit width for spectrophotometry. Since the FLOYDS spectrographs are mounted on the $2 \mathrm{~m}$ telescopes' Cassegrain rotator stages, the slit may be oriented along any desired position angle on the sky. 

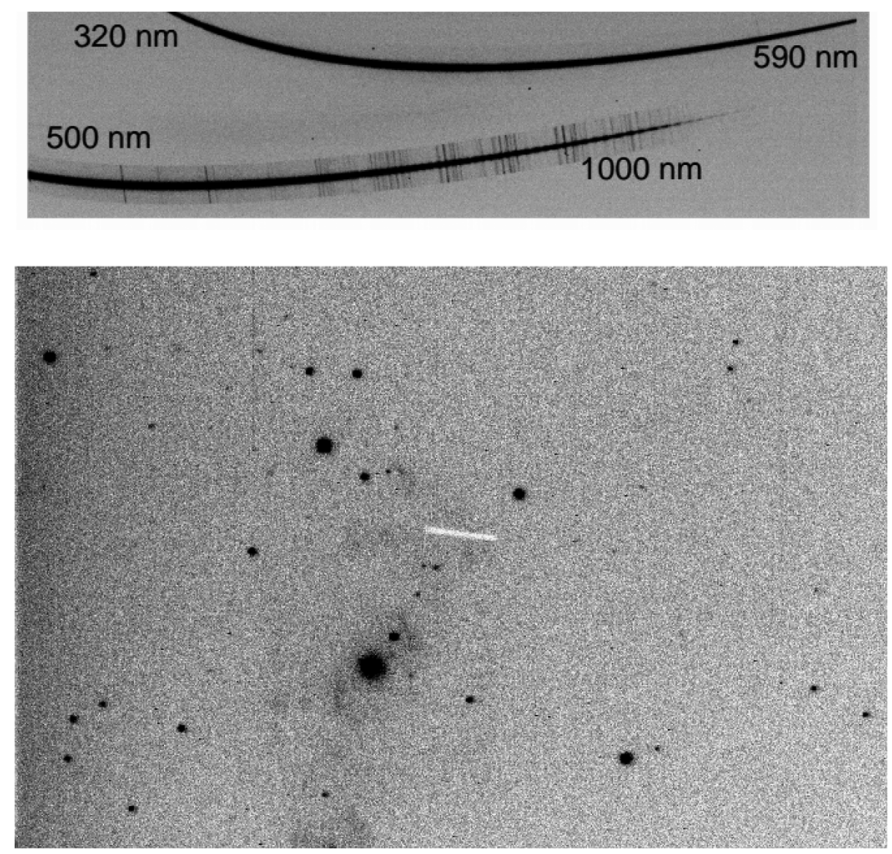

FIG. 6.-Top: A two dimensional FLOYDS spectrum as seen on the CCD. The top trace is second order light, going from 320 to $590 \mathrm{~nm}$, while the bottom trace is 500-1000 nm. Bottom: An image from the FLOYDS slit viewing camera, with its $4 \times 6$ field of view. The slit is easily seen in the center of the image.

There are plans for atmospheric dispersion compensators on each FLOYDS, but these are not yet implemented. An Andor Newton 940 CCD controller package, with an e2V 42-10 CCD ( $13.5 \mu \mathrm{m}$ pixels and a $2048 \times 512$ format), is used with a broadband ultraviolet-enhanced coating. The Andor Newton CCD package is thermo-electrically cooled to $-70 \mathrm{C}$, with negligible dark current. Spectra do fringe above $\sim 700 \mathrm{~nm}$, but this is easily corrected when a flat field is taken at the same telescope orientation as a science frame.

Wavelength calibration is accomplished with a Mercury Argon (HgAr) lamp, and flat fielding with a combination of a tungsten halogen and high-powered xenon lamp (whose light beams are combined with a dichroic). The calibration unit housing these lamps sits in a cabinet near the telescope with a fiber connection to FLOYDS, using a Polymicro FBP broad-spectrum optical fiber for good transmission across the full FLOYDS bandpass. A deployable arm in the FLOYDS instrument can direct light from the calibration unit into the spectrograph, and accompanying optics delivers light with an $\mathrm{f} / 10$ beam, mimicking the actual Faulkes telescopes.

For thermal stability and weight reduction, we adopted a space-frame mechanical structure made of Invar 36, similar to the Levy Spectrometer at the Automated Planet Finder Telescope (Radovan et al. 2010). The four slits are interchanged via a rotary stage; slit position repeatability is better than 0.1 pixels. FLOYDS is extremely stable to thermal changes, and has never been refocused since hardware commissioning, although a stepper motor associated with the collimator is in place in case the need arises.

Robotic acquisition of spectroscopic targets is the key ingredient for making robotic spectroscopy possible. FLOYDS uses a slit-viewing camera and SBIG STL-6303E CCD camera to image a $\sim 4^{\prime} \times 6^{\prime}$ field around the slit both for target acquisition and guiding (Fig. 6, bottom). Automated target acquisition is accomplished via one of three modes: (1) a direct world coordinate system solution of the image with a tailored call to astrometry.net (Lang et al. 2010), followed by centroiding on the object closest to the target's expected position; (2) placing the brightest point source within the telescope pointing error circle $\left(\sim 30^{\prime \prime}\right)$ into the slit (useful, for instance, for standard stars and bright $\mathrm{SNe}$ ); and (3) performing a "blind offset" from a bright star by a set amount, for cases in which the target's absolute position is not well-defined.

The FLOYDS data reduction pipeline is a python/pyraf script that performs standard image detrending (bias subtraction, flat field correction and defringing), spectral extraction, flux and wavelength calibration, and spectral combination of the two orders. In the future, we intend to integrate these procedures more tightly with the LCOGT image-reduction pipeline, and we may implement automated transient classification to shorten the time lag between data taking and classification announcements.

\subsection{NRES}

The Network of Robotic Echelle Spectrographs (NRES) will comprise six identical high-resolution $(R \simeq 53,000)$, precise (radial velocity repeatability $\lesssim 3 \mathrm{~m} \mathrm{~s}^{-1}$ ) optical $(380-860 \mathrm{~nm}$ ) echelle spectrographs, each fiber-fed simultaneously by two $1 \mathrm{~m}$ telescopes and a thorium-argon (ThAr) calibration source. Thus, NRES will be a single, globally-distributed observing facility, composed of six units (one at each of six Network nodes), using $12 \times 1 \mathrm{~m}$ telescopes. NRES will roughly double the RV planetvetting capacity in the USA, and will achieve long-term precision of better than $3 \mathrm{~m} \mathrm{~s}^{-1}$ with exposures of less than an hour for Sun-like stars brighter than $V=12$. Our first spectrograph is scheduled for deployment in 2014 spring, with the full network operation of all 6 units beginning in 2015 fall.

\subsubsection{Spectrograph Design}

The NRES optical design, illustrated in Figure 7, is similar in concept to spectrographs designed for the Palomar East Arm Echelle (Libbrecht \& Peri 1995), the Lick Automated Planet Finder, the Carnegie Planet Finder Spectrograph (Crane et al. 2006), and SOPHIE (Perruchot et al. 2008). The aim of this design is to achieve very high optical throughput, wide wavelength coverage, and simultaneous fiber input from two telescopes. ${ }^{13}$

\footnotetext{
${ }^{13}$ Please see http://lcogt.net/network/instrumentation/nres.
} 


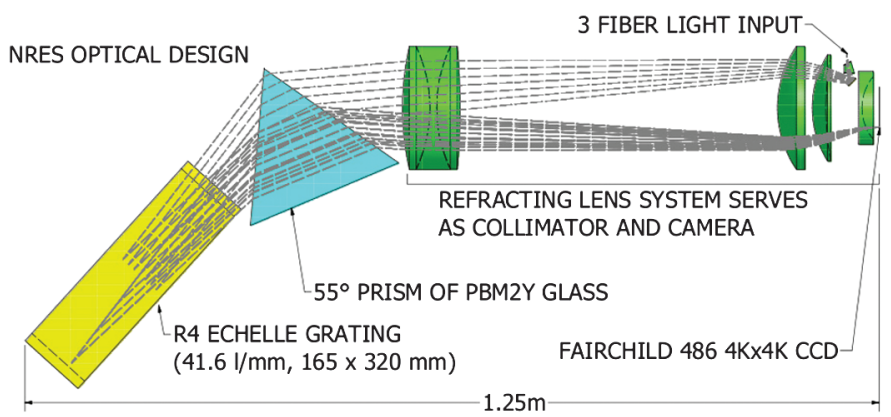

FIG. 7.-Optical Layout of NRES spectrograph. Starlight is injected via a folding mirror at the top right. The field flattener at far right doubles as a window to the CCD dewar.

To maintain wavelength stability, we will place the spectrograph in an environmental chamber that maintains temperature stability of $0.01^{\circ} \mathrm{C}$ and constant barometric pressure within $0.2 \mathrm{mb}$. These limits will assure Doppler stability (uncalibrated against the ThAr wavelength standard) at the level of a few tens of $\mathrm{m} \mathrm{s}^{-1}$, a factor of 10 better than the unavoidable baseline shifts due to Earth's rotation. Only one moving part- the shutter-will reside inside this environmental chamber. By eliminating mechanisms necessary to adjust optics, we simplify the design, and (more importantly) assure a system that has great intrinsic stability. We have tested our shutter design through more than $10^{6}$ open/close cycles without a failure.

For NRES we will use the same CCD controller as for our standard Sinistro Imagers (Tufts et al. 2008), but with a different cryostat and re-tuned analog electronics. The Fairchild 486 $\mathrm{CCD}$ detectors have $4 \mathrm{~K} \times 4 \mathrm{~K}$ format with $15-\mu \mathrm{m}$ square pixels, and are thinned and backside-illuminated, with broadband antireflection coatings. Their quantum efficiency peaks at $91 \%$ at $550 \mathrm{~nm}$. The planned $62.5-\mu \mathrm{m}$ input fibers will be overresolved by a factor of 4.15 , oversampling that is very desirable for precise radial velocity measurement. We expect to achieve a read noise of about $7 e^{-} /$pixel at $1 \mathrm{MHz}$ readout rate, requiring $16 \mathrm{~s}$ to read the full format.

The analysis pipeline will consist of modules for image calibration, spectrum extraction, flux and wavelength calibration, radial velocity determination, stellar classification, quality assurance, and interaction with the data archive. An important goal in this development is to minimize human interaction so that large numbers of spectra can be analyzed in a very short time, and so that access to the data is easy.

\subsubsection{Prototype}

During the time NRES has been under design, we have also assembled a scaled-down prototype, which we are using to reduce risk to the NRES program. The prototype is an $R=25,000$ cross-dispersed echelle with the same basic design as NRES, built mostly from off-the-shelf parts. We have installed it at LCOGT's 0.83-m telescope at BOS (see § 4.5).
The main development goals of the prototype are to expose unanticipated problems in the general spectrograph design via an end-to-end test; validate the design of the Acquisition and Guiding Unit (AGU), which is critical to obtaining high optical throughput; test hardware and software for the spectrograph's environmental control; and provide a realistic testbed for the data analysis software.

We obtained first light with the prototype on the BOS telescope in 2012 October. Final commissioning and regular operation are awaiting completion of a major upgrade of the BOS telescope's control systems. This is due to be finished by 2013 May.

\subsection{Extinction Camera}

Each $1 \mathrm{~m}$ telescope is equipped with an Extinction Camera. This wide-angle optical sky imager is designed to provide the climatic "context" of each observation, i.e., data on cloud coverage, transparency, and extinction. We use Santa Barbara Instrument Group STL-6303E CCD Camera with a format of $3072 \times 20489-\mu \mathrm{m}$ pixels. A filter wheel holds five filters, as noted in Table 3. With a Nikon f/2.8 $400 \mathrm{~mm}$ lens, this camera gives an image scale of $4^{\prime \prime} .64 \mathrm{pix}^{-1}$, and a total field of view spanning $2^{\circ} .6 \times 4^{\circ} .0$. The camera is chilled to $-20^{\circ} \mathrm{C}$ by a Peltier cooler with waste heat dumped to the telescope's liquid cooling system. In dark skies, this system reaches $V=14.9$ in a typical $40 \mathrm{~s}$ integration. We mount the Extinction Camera piggy-backed on the $1 \mathrm{~m}$ telescope primary cell, coaligned with the telescope optical axis with an accuracy of a few tens of arcminutes. The extinction cameras permit rapid comparison of many standard calibrator star magnitudes with those expected in clear, photometric conditions, and hence estimates of the current transparency (Pickles \& Rosing 2013).

\section{SOFTWARE}

In order to simplify development and maintenance, the LCOGT software architecture is highly centralized. The network topology is a "hub and spoke" configuration wherein the organization headquarters near Santa Barbara functions as the hub, radiating control commands to each of the observatory nodes, and pulling back status information and astronomical data. Our software architecture reflects this through the notion of central processing versus processing done at the observatory nodes. Tasks such as observation request handling, telescope scheduling, configuration management, final data reduction, and data archival are handled at headquarters.

Observatory nodes, telescopes, and instruments are treated as stand-alone slaves which must robustly and autonomously execute the instructions they have been given, including operating independently for a period if network connection to the node is lost. Each node must return observation completion status and updates to technical or weather issues that affect the evolving global schedule. Node and schedule monitoring tasks are 
designed to be comprehensive for the automatic systems, and infrequent for humans.

As shown by its reliable operation of eight telescopes at four nodes (including the Back Parking Lot node in Santa Barbara) for several months, the software needed to operate the Network is now mature enough to perform its functions in a workable fashion under most circumstances. Nevertheless, we expect to continue vigorous software development for many years, as all software systems evolve to provide more functionality, better monitoring, better and simpler interfacing between components, fewer failure modes, and better self-correction.

\subsection{Proposal Path}

As with all other aspects of LCOGT operation, the process of accepting, scheduling, queuing, and executing observation requests will be highly automated. The primary point of contact for users of LCOGT will be a web portal. Through the web portal, users will submit proposals for evaluation by the Time Allocation Committee (TAC), view the status of pending proposals, request observations for proposals that have been awarded time, monitor pending observation requests, and access data from observations.

The TAC process will allocate time and assign priorities to each proposal, based on scientific merit. Once a proposal has been awarded time, observation requests (specifying a particular target, generally along with timing or other constraints) can be added to that proposal at any time, for execution on the Network. As observation requests are added to a proposal, they will be digested by the scheduler to be factored into the observing plan, and slated for execution on particular telescopes throughout the network.

When an observation has been obtained, science data will flow through the LCOGT central hub to be calibrated, and then passed on to a science archive (see $\S 6.8$ ) where users can retrieve their data via various web interfaces. Observation status alerts and links to data will be integrated into the web portal for a simple single point of access.

\subsection{Observation Requests and Scheduling}

Perhaps the greatest challenge for the Network design is the need to schedule requests for disparate kinds of observationseach with its own constraints and TAC-assigned priority—so as to maximize LCOGT's scientific productivity. Some aspects of the scheduling operation seem clear. For instance, to be sure that the telescopes will always be kept busy, it is desirable to maintain a varied pool of low-priority observing programs that can be executed in short fragments; in this way the scheduler will always have programs available to fill gaps between higherpriority observations. Similarly, sky flats and other calibration data needed by all users should be scheduled as programs that are owned by LCOGT, and that are automatically placed into the times when they must occur, at high priority. Such strategic choices do not, however, speak to the tactical choices that a scheduler must make to interleave programs with complicated temporal constraints into a whole that makes best use of the observing resources. Indeed, a central part of the problem is to define what is meant by "best use" in this context. Also, the nature of "best" algorithms and their outcomes will depend on the mix of observation types desired by users-a mix that is difficult to predict and that is likely to evolve in time. For these reasons, we expect that scheduler development will continue as a major activity at LCOGT for the foreseeable future, and we have so far eschewed predictions of the scheduler's efficiency. In the following paragraphs we therefore describe the scheduler's appearance to the user in some detail, but we say little about specific algorithms used in it (by design, these are modular and changeable), or about its current performance.

In LCOGT's terminology, the minimum schedulable unit of the Network is the block. Each block is bounded in time, and consists of a number of molecules, defined as one or more exposures with a single instrument configuration, at a single telescope pointing. Multiple molecules may be grouped within a single block. This structure allows both the user and the network scheduler to operate at a level above raw exposures to define a useful unit of work in the context of any particular proposal.

Observation requests must include target coordinates and filters, and may include constraint specifications such as seeing, transparency, airmass, lunar phase and angle. Requests have a priority assigned by a TAC. High priority requests, including target of opportunity observations, can pass quickly though the portal and scheduler to a node and telescope, possibly interrupting previously scheduled observations. An interrupted block may resume automatically if the interruption is short, or may be rescheduled.

Users specify observation requests in a flexible grammar that allows arbitrary groupings of individual observations into compound requests for evaluation by the network scheduler. For example, a user conducting periodic monitoring of a variable source may specify a series of observations of a common target at specific times or with approximate spacings in time, and group these observations together as a single compound request. This says to the scheduler that all the observations in the compound request must be obtained in order to satisfy the request. The web portal provides a graphical environment for composing such requests, but ultimately requests are transferred in a machine-readable format (JavaScript Object Notation), which provides for programmatic submission as well.

Observation requests may be submitted to the network either manually via the web portal or programmatically via an application program interface layer. These submissions are stored in the Request Database, where they become visible to the network scheduler. As requests are processed and observations obtained, their status in the request database is updated, and fed back to the user via the web portal. 
The network scheduler is responsible for taking observation requests from the request database and distributing them as discrete observations to be made at specific times on specific telescopes. Observation requests may be simple or complex, specific or highly abstract, and may consist of multiple discrete observing units chained together. In order to allocate observations to the Network, the scheduler must consider such factors as program priority, observing constraints, resource availability, and changing weather conditions. With these constraints in hand, the scheduler takes the set of incomplete requests and uses them to produce a schedule of blocks for each telescope.

\subsection{Proposals and Observations Network Database (POND)}

Once a schedule has been produced, the corresponding blocks are stored in the Proposals and Observations Network Database (POND). This database acts as the transport layer between the network scheduler and the Network nodes. Blocks are picked up from the POND by each site agent and handed to their respective telescopes for execution. Since blocks are the smallest schedulable unit, all molecules within a block must succeed for the block to be counted as successful. As the status (successful or unsuccessful) of scheduled blocks becomes known, they are updated in the POND, allowing the scheduler to update the execution of the schedule. The schedule may be dynamically recalculated as necessary, in response to block status information, to accommodate new requests, or to handle changing network state such as weather conditions or technical failures.

\subsection{Node Software}

Observations arrive at nodes for execution by a "pull" mechanism. The Site Agent at each node is responsible for checking the POND and pulling down the node-schedule. This update happens regularly as long as network communications between the node and the POND are good, but in the event of a network outage, the site agent maintains a cache of the latest schedule so it can continue to execute for up to $72 \mathrm{hr}$ without outside connection.

The site agent pushes individual observations to a sequencer agent for each telescope when conditions are correct for executing the next block in the schedule. The sequencer controls all aspects of the telescope, instrument, and enclosure necessary to accomplish the observation. It manages a large dependency tree to ensure all systems are in proper states for any given observation before data are collected. Observations result in the creation of data artifacts which are analyzed in real time, and success or failure of the observation is communicated back to the site agent. Ultimately, the site agent pushes observation status back to the POND so that the scheduler can perform bookkeeping and revise the schedule as necessary.

Each component of the system constantly collects telemetry data, which accumulate in a database at site. These data can be graphed on the engineering interface to facilitate immediate or historical diagnoses of events at site in fine detail. These telemetry data are subsequently resampled into coarser detail and replicated back to a central telemetry database for performance analysis over longer time periods.

\subsection{Telescope Control System}

There are no particularly new technologies in the design of our telescopes, but their control software assumes a very high level of reliable, autonomous behavior.

The software architecture at each telescope node (controlling the node, weather and safety systems, enclosures, telescopes, and instruments) comprises a Java-based telescope control system (jTCS) incorporating a loose collection of many semiautonomous agents, developed in the Java Agent DEvelopment framework (JADE). Each agent is responsible for a small part of the overall control and monitoring system, and all participate in a publish and subscribe (PubSub) system to share data and send each other messages. This distributed architecture simplifies the overall control semantics and makes it more robust against failures: If any agent dies, the remainder of the system can continue to operate and attempt recovery of failed components.

The main agents for each telescope include (1) an Astrometric and guiding agent to configure multiple instruments in the focal plane, based on Tpoint and the TPK kernel (Terrett 2006); this uses Astrometry.Net for automatic WCS fitting and to place and guide spectroscopic targets; (2) axis control agents to servo the telescope to the latest target coordinates; (3) agents to monitor International Earth Rotation and Reference Systems Service (IERS) bulletins and to configure each telescope and instrument in the focal plane; and (4) agents to control all enclosure and telescope systems. Guiding corrections can come from any instrument, including spectrograph slit-viewers, or the science instrument itself.

LCOGT has developed a comprehensive embedded mechanism control system based on the Blackfin microprocessor family. This system enables internet control of motors, fans, dry-air systems, mechanisms such as focus, collimation, filter wheels, mirror covers, and sensors such as temperature and position probes. The Blackfin architecture also enables us to design "smart" power modules to support power cycling and current monitoring of each subsystem, via a JADE agent. These are key components of the telescope system's ability to autonomously recover from errors and outages.

Each $1 \mathrm{~m}$ telescope provides support for up to four cooled instrument electronics crates below each mirror cell for control of all instrumentation, fans, sensors, and monitoring equipment. The $0.4 \mathrm{~m}$ telescopes support similar functionality with fewer control modules, but with identical servo mechanisms. Our $2 \mathrm{~m}$ telescopes are in the process of off- and on-mount control systems upgrades, so they too can be integrated into this PubSub agent control system and become nodes of our global network. 


\subsection{Configuration Database}

For a network as large as LCOGT's, the number of items such as telescopes, mirrors, cameras, and filters that are deployed in the field and that could potentially impact data quality and provenance runs into the thousands. In order to tackle this problem of asset management, we have created a database system, called the ConfigurationDB (ConfigDB) using the Django web framework.

The ConfigDB records details of the sites, enclosures, telescopes, instruments, cameras, filters, and also several wavelength-dependent quantities such as filter transmissions, CCD QE curves, and mirror reflectivities. It also stores start and end dates of operation, status, and other data relevant for all of these items. Website and programmatic interfaces allow users to find the canonical details on the equipment used to take their data, and to perform tasks such as automatically producing filter transmission plots.

\subsection{Data Pipelines}

A large network of telescopes such as LCOGT's that will be used for a very diverse set of scientific goals raises unique challenges that are not present in a single-purpose survey or traditional common-user facility. The large number of instruments and the volume of data they will generate means that LCOGT, as the data originator, is in the best position to understand and to reduce the data optimally. On the other hand, the wide variety of scientific programs that will be running on the network, and their diverse needs for data reduction, renders it almost impossible to make a generalized pipeline optimal for all potential science needs.

Accordingly we have designed the pipeline with the philosophy of doing the best we can for the bulk of potential users, and making the pipeline products that are of the most general use. At the same time, we aim to avoid controversial steps in the data reduction that could be problematic for end users of the products or to attempt to do the end-users' science for them. In addition, the pipeline emphasizes recording of the processing steps performed, the parameters used, and the software versions employed. These steps are of vital importance for traceability of the reduced data, and to document its provenance. The topic of provenance is of increasing importance as astronomical data sets grow in size and the degree of separation between the data producer and user increases (e.g., Berriman \& Deelman 2009).

To provide a pipeline that can handle the diverse instruments of the LCOGT network, facilitate adding new instruments, and allow changes to the type of data products to be made, we need a generalized infrastructure that supplies these capabilities. This generic infrastructure is supplied by ORAC-DR (Cavanagh et al. 2008,2003 ) which was originally written to support SCUBA at the JCMT but has been extended and generalized (e.g., Currie
2004) to support a wide variety of instruments and observing types.

The pipeline is entirely data-driven and requires no user input. Processing is controlled by modular recipes defining the steps necessary to reduce the data. Each recipe is a list of data reduction steps to perform on each frame or group of frames. These individual steps are known as primitives, and each primitive performs one astronomically-significant step such as dark subtraction or source catalog production. As most of the data reduction steps are common across classes of instruments, a small set of primitives is sufficient for the majority of processing needed in the LCOGT pipeline.

The main recipes currently in use handle the combining of raw bias, dark and flat frames into master calibration frames and the processing of regular science frames to produce the $\mathrm{BCD}$ (Basic Calibrated Data) products. For these science frames we perform the following operations:

1. Bad-pixel masking

2. Bias subtraction

3. Dark subtraction

4. Flat field correction

5. Astrometric solution

6. Source catalog production

7. Zeropoint determination

8. Per-object airmass and barycentric time correction computation

We perform bad-pixel masking, bias and dark subtraction, and flatfield correction in the normal manner, using the ORAC-DR calibration infrastructure to select the nearest (in time) calibration frame that satisfies the constraints of binning, filter, etc. For astrometric solution, we use autoastrom against the UCAC3 catalog (for $1.0 \mathrm{~m}$ and $0.4 \mathrm{~m}$ data; Zacharias et al. [2010]) or the Tycho-2 catalog (for Context Camera data; Høg et al. [2000]). Source catalogs are produced using the SExtractor software (Bertin \& Arnouts 1996) to perform object detection, source extraction and aperture photometry. In total, we output 49 parameters for each detected source, consisting of positional information along with its estimated errors, and information on the shape and extent and the measured flux and flux error in four fixed and two variable apertures. Following pipeline processing, the $\mathrm{BCD}$ products consisting of the reduced images and source catalogs, PNG bitmap versions of the images, and nightly and data quality control logs are transferred to the science archive for ingestion and distribution.

\subsection{Science Archive}

The science archive is expected to play a central role in the LCOGT network, as this is the primary means for scientists and other users to get access to the data taken by the network. As the public face of the data store, it is intended to be accessible and intuitive for a wide variety of potential users. 
The science archive is being built by IPAC ${ }^{14}$ based on a software and hardware architecture that were developed for the Keck Observatory Archive (KOA) and the NASA Star and Exoplanet Database (NStED) (Berriman et al. 2010).

The archive checks and verifies batches of BCD products (processed images and their metadata, source catalogs, photometric data, and frame bitmaps) from the pipeline upon reception and then, if they pass these checks, ingests them into a relational database management system to allow fast and efficient querying. In addition, ancillary data products such as the processing logs and master calibration files contained within a data batch are stored and indexed for potential retrieval.

Data from science programs can be read only by authorized users during a proprietary period which defaults to one year; data owners may also choose a shorter period, or decide to waive the proprietary period. Data taken for educational purposes are immediately available to all users.

One can access the science archive both via a graphical webbased interface that allows sophisticated searching, filtering and plotting tasks, as well as via a programmatic interface. This programmatic interface will also be used to integrate archive queries and functionality into applications developed and hosted by LCOGT such as those for public education and Citizen Science (see $\$ 8$ ). Since the main Archive does not store raw data images, these images are available to users on request, through LCOGT servers operating at our Santa Barbara headquarters.

\section{ASSEMBLY AND DEPLOYMENT}

Time-domain observing places unusually stringent demands on obtaining reliable and consistent behavior from the Network's components. To meet these demands, we adopted an approach in which LCOGT itself carried out almost all of the major development tasks. Thus, most major system components (site development, enclosures, telescopes, instruments, computing hardware and software) were designed, fabricated, integrated, tested, and deployed by LCOGT personnel.

\subsection{Prototyping}

We make heavy use of prototypes in a design-fabricateintegrate-test-redesign cycle to enable full unit and integration testing before major Network units leave the factory floor. At an early stage in the design process for each LCOGT major system, a collaborating team of engineers develop a prototype. This team unit-tests each subunit to ensure components are appropriate for the demands of a fully remote and robotic system, to set control sequences and limits, route and protect cabling, manage thermal outputs, streamline manufacturing procedures, and ensure performance and function. When the prototype functions satisfactorily, it is integrated into the full test observatory system

\footnotetext{
${ }^{14}$ Please see http://www.ipac.caltech.edu.
}

which has been configured at the headquarters near Santa Barbara, in a site affectionately known as the Back Parking Lot or BPL. Integration testing places the prototype into the full observatory context with current software, enclosure, and other equipment and services. The team can then evaluate and improve the installation and servicing procedures, minimize thermal footprints, and route cabling. They then run the devices through test sequences that replicate normal operations and, where appropriate, operate the observatory on-sky to ensure design specifications are met for alignment, optics, imaging, and performance. When the prototype is finalized, it is typically upgraded to the latest revision and then used as a test device at headquarters to help locate and manage any problems encountered with production devices later on. The team updates detailed plans and bills of materials, and stores them in our version-controlled, parts management system from Arena Solutions. Purchasing accesses the latest revisions from these systems and the Logistics and Telops Teams maintain a stock of spares of key parts and assemblies, both at headquarters and on-site. Last, we push any design modifications back into Arena and issue change orders as needed for production devices.

\subsection{Assembly}

Once a prototype has been proven in the full observatory, the development team reviews the plans and signs off on them. The team then has an adequate number of parts machined, fabricated, or purchased. Where necessary, the full anticipated build-out of parts (e.g., mirror sets for $15 \times 1-\mathrm{m}$ telescopes, filter sets, CCDs, etc.) is acquired to ensure manufacturing consistency and lower cost. Normally, astronomical telescope developments are one-off efforts; in such cases this elaborate prototype and documentation strategy would not be cost-effective. But to produce a dozen or so identical units for a network, procedures like these are not only essential for correct performance, they also offer economies of scale that significantly lower overall cost.

We build all observatory components, including the IT support building and the enclosures, at LCOGT headquarters in one of four assembly bays-enclosures, electrical panels, $0.4 \mathrm{~m}$ telescopes, and $1 \mathrm{~m}$ telescopes. We run full unit tests on each major component and integrate the primary systems-computing, electrical, and cooling_-prior to breakdown for shipment. Each item is then broken down into its major components for shipping to site. The assembly crew consists of a project engineer and telescope technicians working closely with the design engineers. The participation of project engineers ensures that nuances of alignment, calibration, assembly, and cabling are communicated and documented.

\subsection{Deployment}

Site and observatory development follow a standard sequence. We first complete site civil engineering, including concrete and conduit work. For the 1-meter telescopes, we then ship 
the Ash-domes and enclosure walls to site and assemble them. In the next stage, we install the electrical and computing systems. This includes the site IT rack, the telescope and dome control panels, the weather station, the air conditioning units, and the site air compressor. We then ship the telescopes to site, reassemble, integrate, align and collimate them, and test them on sky. One of the project engineers responsible for final assembly and testing at headquarters travels with a team of engineers and telescope technicians to site for the installations. The $0.4 \mathrm{~m}$ telescopes, when they are deployed, will follow a similar site installation sequence.

Due to the extensive prototyping and testing at headquarters, first light on $1 \mathrm{~m}$ telescopes has typically been achieved within a week of installation. Robotic control is possible within an even shorter period. During the six months following first light, we combine engineering evaluation with internal science use to validate the overall system.

\subsection{Telops}

Telescope operations (Telops) for a global robotic system is a critical factor in maximizing the available observing resources and in maintaining cost-effective observatory operations. LCOGT maintains an experienced crew of Telops experts in the UK, California, Hawaii, and Australia. Moreover, Network nodes are located only at established observatory sites, where routine maintenance and noncritical service or replacement tasks can be completed by local staff on a contractual basis. We store a reasonable stock of spare parts, tools, and other necessary gear (such as scaffolding) at each site. Finally, we take pains to identify performance problems early, through a comprehensive set of software agents that provide status, error reporting, and direct observation and control of these remote facilities.

\section{EDUCATION AND OUTREACH}

The aim of the LCOGT education and outreach program is to excite, inspire and encourage learners of all ages to pursue science investigations and develop their critical thinking skills. We are doing this by constructing programs that immerse educational users of our telescopes in fully-formed observational projects that can engage users at many levels of commitment and sophistication. A typical project takes participants through observation planning and scheduling, image inspection, processing, and analysis, and publication of the results, either on our website or to an external organization, e.g., the Minor Planet Center. We approach these goals in three ways: by interacting with individual participants, with organizations, and by creating and distributing web-based resources.

\subsection{Citizen Science}

Citizen Science is often synonymous with "crowd sourcing", where many people repeat a trivial activity a small number of times to produce a more accurate result than a small number of people doing the same many times. Citizen Science can take this idea further, allowing lay-people to participate in scientific investigation without specialized knowledge or experience, but training them to think like scientists by having them go beyond mere acquisition of data to a meaningful analysis of the results.

As a pilot to this citizen science approach we created Agent Exoplanet, ${ }^{15}$ a browser-based application which guides participants as they perform photometry on selected exoplanet transit images, produce lightcurves, and draw basic conclusions from these data. We are currently evaluating this resource and considering which other areas of astronomy are adaptable to this approach to make the most effective use of our telescope network with our growing cadre of citizen scientists.

Key to these citizen science projects is the idea that an individual can make a contribution to the project, however great or small, which shapes the overall outcome. In Agent Exoplanet each person can analyze image data, but this analysis is then pooled to produce a master lightcurve for each exoplanet transit. This fosters the idea that not only is an individual's contribution valuable, but that each person's best effort when analyzing the data improves the end result for all.

\subsection{Education and Citizen Science Partners}

As part of the LCOGT citizen science program we will support a small number of projects such as Agent Exoplanet at any one time. However, we will also encourage external groups and organizations to make proposals to our time allocation committee for new educational or citizen science projects. This will be a parallel route to the science proposal process, in which proposals will be judged on their educational and/or scientific merits, how well they make use of the global nature of our network, and how they will support their user base. Our present plans call for dedicating about $10 \%$ of the observing time on the $2 \mathrm{~m}$ telescopes to education projects, a smaller but as yet undefined fraction of the $1 \mathrm{~m}$ time, and $50 \%$ of the time on the $40 \mathrm{~cm}$ telescopes. Individuals wanting to use our network will be able to sign up either to one of the LCOGT programs or to a program supported by external organizations, depending on the type of observing experience they desire.

Currently we are working with three organizations, our pilot education partners, in their use of our expanding telescope network. Each of these organizations, the Faulkes Telescope Project (UK), University of Hawaii astronomy outreach program (US), and Space to Grow (AU), support distinct educational programs using our telescope resources. We have worked with these education partners on teacher training programs, partnering schemes between astronomers and schools, and carried out scientific investigations monitoring asteroids, NEOs, and supernovae.

\footnotetext{
${ }^{15}$ Please see http://lcogt.net/agentexoplanet.
} 
We are focusing effort on making our telescope resources versatile with intuitive, web-based observation requesting and simple data analysis tools. By making these resources available to education organizations to run their own programs, LCOGT can have a broader and more sustainable reach in the community than if we were to run all programs in-house.

\subsection{Online Astronomy Resources}

In addition to creating programs centered around astronomical observing and data analysis, an important aspect of our education program is to provide web-based tools to help people explore astronomy. The following three are a selection of our most popular resources, which have been used by school children and teachers, by amateur astronomers, and by the general public to enrich their experience of astronomy.

Star in a Box explores the lifecycle of stars, a common topic in school curricula. We developed this interactive HertzsprungRussell diagram ${ }^{16}$ as a resource for teachers wanting extra support when teaching this topic.

SpaceBook is an online astronomy textbook written by us. It covers most aspects of astronomy with insights into areas of expertise provided by our science and technical teams. ${ }^{17}$

Virtual Sky is a planetarium web application which can be embedded into any website. We developed this to be easily customizable for viewing location, sky projection and many other features. ${ }^{18}$

Full details of our education and citizen science programs can be found on our website: http://lcogt.net/education.

\section{FIRST SCIENTIFIC RESULTS}

On the night of 2012 April 29, less than one month after first light, we used the first McDonald $1 \mathrm{~m}$ telescope to obtain a light curve of a new white-dwarf-containing binary showing deep eclipses. We used no filter in order to gain sensitivity, with an exposure time of $60 \mathrm{~s}$ and a total cycle time of $75 \mathrm{~s}$. The light curve is shown in Figure 8, displayed as relative flux vs. time. The object was not detected on the CCD during eclipse, so the in-eclipse measurements are marked by the 3-sigma upper limit on the CCD sensitivity. The left panel of Figure 9 shows an outof-eclipse image centered on the target, while the right panel shows an in-eclipse image, where the target cannot be seen.

The duration of the eclipse, combined with multi-band light curves obtained at other LCOGT telescopes, indicate the companion is a low-mass star. Therefore this system is a possible pre-CV, post common envelope eclipsing binary. A full analysis of all data obtained for this object will be presented elsewhere.

These observations were part of a LCOGT photometric follow-up of deeply eclipsing WD candidates, with the goal

\footnotetext{
${ }^{16}$ Please see http://lcogt.net/education/starinabox.

${ }^{17}$ Please see http://lcogt.net/spacebook.

${ }^{18}$ Please see http://lcogt.net/virtualsky.
}

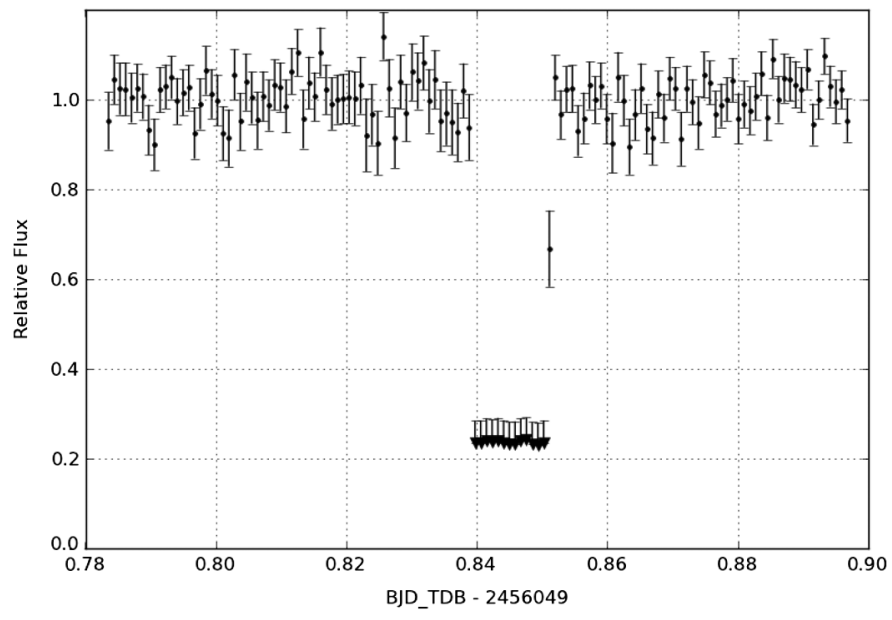

FIG. 8.-White dwarf light curve taken at the ELP $1.0 \mathrm{~m}$. Observations were done with no filter and the exposure time was $60 \mathrm{~s}$. The in-eclipse measurements are upper limits as the object was not detected on the CCD in those exposures.

of detecting WDs orbited by cold objects, including low-mass stars, brown dwarfs, and planets down to sub-Earth size. This project is motivated by detections of close-in substellar companions to WDs (Maxted et al. 2006; Littlefair et al. 2006), and many other observations suggesting the existence of planets around WDs (e.g., Farihi et al. 2009; Debes et al. 2012). The large amount of LCOGT telescope time and future technical capabilities, including the network mode, will be valuable in helping to quickly and efficiently confirm and characterize deeply eclipsing WD candidates and to characterize their substellar companion population, in turn constraining scenarios for binary system and planetary system evolution beyond the main sequence (e.g., Nordhaus et al. 2010).

\section{SUMMARY}

In 2012, LCOGT began its transition from designing and fabricating a global telescope network, to operating it and using it for science. Although the $1 \mathrm{~m}$ Network will not be complete (through Phase B, as defined in $\S 2$ ) until the end of 2013, it

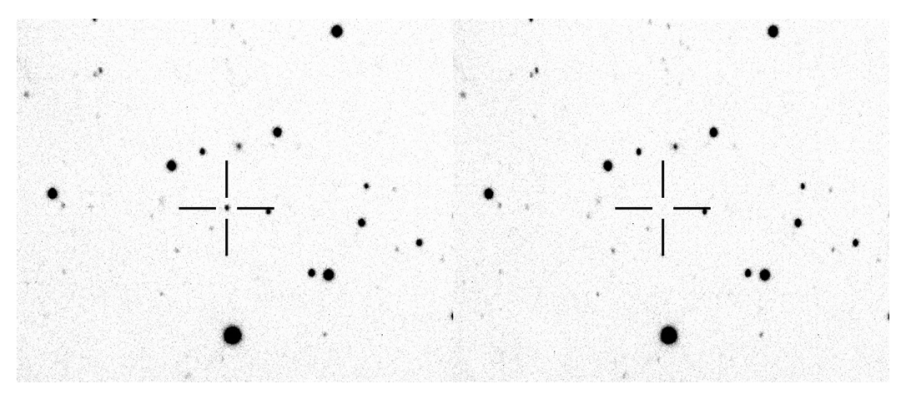

FIG. 9.-Out-of-eclipse (left) and in-eclipse (right) imaging of the white dwarf vicinity, done at the ELP $1.0 \mathrm{~m}$. The object was not detected on the CCD in the in-eclipse exposures. 
is clear already that the Phase-B Network, comprising a full ring of nodes in the southern hemisphere and two nodes (plus additional facilities) in the north, will enable new kinds of time-series astronomical observations, as intended. This network of $10 \times$ $1 \mathrm{~m}$ telescopes is supplemented by new low-resolution spectrographs on the Faulkes $2 \mathrm{~m}$ telescopes, by a number of $40 \mathrm{~cm}$ imaging telescopes at sites worldwide, and (in 2014) by radialvelocity-capable echelle spectrographs on the $1 \mathrm{~m}$ telescopes. This paper documents these facilities.

Looking forward, many challenges remain for LCOGT and the Network. On the level of facilities and instrumentation, we still hope to complete a northern ring of $1 \mathrm{~m}$ telescopes to match the southern one (the Phase-C deployment). Achieving this will require funding from new sources, perhaps including raising funds from new partners in the Network. Using the Network efficiently will demand scheduling and other software support that goes significantly beyond the expected capabilities of our first-generation scheduler, proposal-tracking tools, and other operational facilities. Thus, we expect to maintain a heavy level of software effort for the foreseeable future. Finally, we must organize the scientific collaborations necessary to apply this powerful observational facility in the most direct possible way to important scientific problems. Through the early stages of developing the Network, we were guided in such matters by our scientific advisory committee, a rotating group of outstanding astronomers from outside LCOGT. Now that full operations are imminent, we must seek a more direct connection with our scientific and organizational partners. We began this process in 2013 January, with the first meeting of the LCOGT Scientific Collaboration, which will govern the use of most of the Network's observing time.

LCOGT's larger transition-from an organization that is mostly concerned with developing a facility to one that is mostly concerned with operations and science-will require significant changes in LCOGT's internal structure, and in its modes of interaction with the astronomical community. These changes are already underway; we will describe them elsewhere.
We are grateful to all who have contributed over the last six years to the success of the LCOGT Network. These include those who have served on our governance and advisory committees: C. Aerts, J. Beckers, T. Bedding, L. Bildsten, J. Bloom, J. Farrell, L. Hillenbrand, D. Largay, T. Marsh, M. Phillips, D. Pollacco, D. Queloz, P. Roche, D. Sasselov, M. Skrutskie, D. Soderblom, N. Suntzeff, J. Tonry, and V. Trimble. We also thank all of LCOGT's employees, past and present, whom we have not named elsewhere: J.R. Aquino, J.D. Armstrong, I. Baker, J. Barton, O. Barton, M. Becker, E. Bildsten, J. Bowen, J. Bower-Cooley, N. Brooks, H.E. Brown, H.J. Brown, J. Brown, N. Brunner, S. Cook, V. Corris, R. Cutler, A. Dai, C. Daniel, D. Mans, B. Donnelly, N. Fairhurst, L. Farinpour, C. Ferrer, A. Fox-Leonard, W. Giebink, C. Gillmore, M. Giorgio, V. Gorbunov, S. Growney, J. Harmon, S. Hausler, N. Hawes, R. Hayes, W. Haynes, A. Henry, I. Henry, S. Hillberry, D. Huthsing, D. Jahng, B. Janus, M. Jeffus, D. Johnson, D. Kent, C. Kim, D. Kudrow, A. Kushnick, P. LaCorte, S. Lad, M. Lagana, K. Lessel, K. Lopker, S. Lowe, M. Loyley, J. Macassey, A. Mansfield, J. McCammon, L. McCormack, G. MedranoCerda, P. Michell, J. Minc, D. Muecke-Herzberg, D. Murray, G. Nelson, D. Norton, A. Ouellett, G. Paredes, C. Phung, A. Piascik, R. Qadri, P. Rees, A. Reiner, S. Ridgeway, G. Rives-Corbett, G. Robertshaw, P. Robinson, M. Royster, N. Ruvalcaba, O. Saa, G. Sandoval, N. Schauser, L. Seale, G. Shannon, J. Shaw, B. Sheppard, L. Simcock, T. Simmons, A. Sinclair, M. Smith, N. Su, S. Taylor, A. Tekola, J. Templeton, D. Thomson, L. Tinajero, J. Towsley, S. Valenti, B. Vanderhyden, J. VanLeyen, R. Welsh, O. Wiecha, K. Wojcik, and C. Wood. We are grateful to Keith Horne, Martin Dominick, their colleagues at St. Andrews University, and to the Scottish Universities Physics Alliance (SUPA) for their generous support in helping to install telescopes in the southern hemisphere. Funding for the NRES spectrographs was provided through MRI Grant AST-1229720 from the National Science Foundation (NSF). We are also grateful to Dr. Grant Williams, who refereed this paper, for his careful reading and helpful comments about the manuscript.

\section{REFERENCES}

Akerlof, C., Amrose, S., Balsano, R., Bloch, J., Casperson, D., Fletcher, S., Gisler, G., Hills, et al. 2000, AJ, 119, 1901

Berriman, G. B., Ciardi, D., Abajian, M., Barlow, T., Bryden, G., von Braun, K., Good, J., Kane, S., et al. 2010, in ASP Conf. Ser. 434, Astronomical Data Analysis Software and Systems XIX, ed. Y. Mizumoto, K.-I. Morita, \& M. Ohishi (San Francisco: ASP), 119

Berriman, G. B., \& Deelman, E. 2009, in Proceedings of the special session "Accelerating the Rate of Astronomical Discovery" of the 27th IAU General Assembly, 2009 August 11-14, Rio de Janeiro, Brazil, http://pos.sissa.it/cgi-bin/reader/conf.cgi?confid=99

Bertin, E., \& Arnouts, S. 1996, A\&AS, 117, 393

Bischoff, K., Tuparev, G., Hessman, F. V., \& Nikolova, I. 2006, Proc. SPIE, 6270, 6270Q
Bloom, J. S., Starr, D. L., Blake, C. H., Skrutskie, M. F., \& Falco, E. E. 2006, in ASP Conf. Ser. 351, Astronomical Data Analysis Software and Systems XV, ed. C. Gabriel, C. Arviset, D. Ponz, \& S. Enrique (San Francisco: ASP), 751

Cavanagh, B., Hirst, P., Jenness, T., Economou, F., Currie, M. J., Todd, S., \& Ryder, S. D. 2003, in ASP Conf. Ser. 295, Astronomical Data Analysis Software and Systems XII, ed. H. E., Payne, R. I., Jedrzejewski, \& R. N. Hook (San Francisco: ASP), 237

Cavanagh, B., Jenness, T., Economou, F., \& Currie, M. J. 2008, Astron. Nachr., 329, 295

Charbonneau, D., Brown, T. M., Latham, D. W., \& Mayor, M. 2000, ApJ, 529, L45

Chromey, F. R., \& Hasselbacher, D. A. 1996, PASP, 108, 944 
Crane, J. D., Shectman, S. A., \& Butler, R. P. 2006, Proc. SPIE, 6269, 626931

Currie, M. J. 2004, in ASP Conf. Ser. 314, Astronomical Data Analysis Software and Systems (ADASS) XIII, ed. F. Ochsenbein, M. G. Allen, \& D. Egret (San Francisco: ASP), 460

Debes, J. H., Kilic, M., Faedi, F., Shkolnik, E. L., Lopez-Morales, M., Weinberger, A. J., Slesnick, C., \& West, R. G. 2012, ApJ, 754, 59 Dubberley, M. A. 2010, Proc. SPIE, 7739, 11

Elliot, J. L. 1979, ARA\&A, 17, 445

Elliot, J. L., Person, M. J., Zuluaga, C. A., Bosh, A. S., Adams, E. R., Brothers, T. C., Gulbis, A. A. S., Levine, S. E., et al. 2010, Nature, 465,897

Farihi, J., Jura, M., \& Zuckerman, B. 2009, ApJ, 694, 805

Filippenko, A. V. 1997, ARA\&A, 35, 309

Fraser, S., \& Steele, I. A. 2004, Proc. SPIE, 5493, 331,

Garrel, V., Guyon, O., \& Baudoz, P. 2012, PASP, 124, 861

Gehrels, N., Ramirez-Ruiz, E., \& Fox, D. B. 2009, ARA\&A, 47, 567

Grundahl, F., Arentoft, T., Christensen-Dalsgaard, J., Frandsen, S., Kjeldsen, H., \& Rasmussen, P. K. 2008, J. Phys. Conf. Ser., 118, 012041

Haldeman, B. J., Haynes, R. M., Posner, V., Tufts, J. R., Pickles, A. J., \& Dubberley, M. A. 2010, Proc. SPIE, 7739, 15

Hawkins, E., Baliber, N., Bowman, M., Brown, T., Burleson, B., Foale, S., Ford, M., Lister, T., et al. 2010, Proc. SPIE, 7737, 7

Hirsch, M., Harmeling, S., Sra, S., \& Schölkopf, B. 2011, A\&A, 531,A9

Høg, E., Fabricius, C., Makarov, V. V., Urban, S., Corbin, T., Wycoff, G., Bastian, U., Schwekendiek, P., et al. 2000, A\&A, 355, L27

Lang, D., Hogg, D. W., Mierle, K., Blanton, M., \& Roweis, S. 2010, AJ, 139, 1782

Law, N. M., Mackay, C. D., \& Baldwin, J. E. 2006, A\&A, 446, 739

Libbrecht, K. G., \& Peri, M. L. 1995, PASP, 107, 62

Littlefair, S. P., Dhillon, V. S., Marsh, T. R., Gänsicke, B. T., Southworth, J., \& Watson, C. A. 2006, Science, 314, 1578

Lohmann, A. W., Weigelt, G., \& Wirnitzer, B. 1983, Appl. Opt., 22, 4028

Maxted, P. F. L., Napiwotzki, R., Dobbie, P. D., \& Burleigh, M. R. 2006, Nature, 442, 543
Mayor, M., \& Queloz, D. 1995, Nature, 378, 355

Mottram, C. 2006, Astron. Nachr., 327, 806

Nather, R. E., Winget, D. E., Clemens, J. C., Hansen, C. J., \& Hine, B. P. 1990, ApJ, 361, 309

Nordhaus, J., Spiegel, D. S., Ibgui, L., Goodman, J., \& Burrows, A. 2010, MNRAS, 408, 631

Park, H.-S., Ables, E., Barthelmy, S. D., Bionta, R. M., Ott, L. L., Parker, E. L., \& Williams, G. G. 1998, Proc. SPIE, 3355, 658

Perruchot, S., Kohler, D., Bouchy, F., Richaud, Y., Richaud, P., Moreaux, G., Merzougui, M., Sottile, R., et al. 2008, Proc. SPIE, 7014, 12

Pickles, A., Rosing, W., Brown, T. M., de Vera, J., Dubberley, M., Haldeman, B., Hausler, S., Haynes, R., et al. 2010, Proc. SPIE, 7733, 5

Pickles, A. J., \& Rosing, W. E. 2013, ArXiv e-prints 1301.3926, http:// adsabs.harvard.edu/abs/2013arXiv1301.3926P

Pickles, A. J., Rosing, W., Martinez, J., Fulton, B. J., \& Sand, D. 2012, Proc. SPIE, 8444, 6

Radovan, M. V., Cabak, G. F., Laiterman, L. H., Lockwood, C. T., \& Vogt, S. S. 2010, Proc. SPIE, 7735, 13

Ricker, G. R., Latham, D. W., Vanderspek, R. K., Ennico, K. A., Bakos, G., Brown, T. M., Burgasser, A. J., Charbonneau, D., et al. 2010, in Bulletin of the American Astronomical Society 42, American Astronomical Society Meeting Abstracts no. 215, 450.06

Saunders, E. S., Naylor, T., \& Allan, A. 2008, Astron. Nachr., 329, 321

Stelzer, C., \& Ruder, H. 2007, A\&A, 475, 771

Terrett, D. L. 2006, Proc. SPIE, 6274, 31

Tufts, J. R., Lobdill, R., Haldeman, B. J., Haynes, R., Hawkins, E., Burleson, B., \& Jahng, D. 2008, Proc. SPIE, 7021, 12

Vanderspek, R. K., Ricker, G. R., \& Doty, J. P. 1992, in ASP Conf. Ser. 34, Robotic Telescopes in the 1990s, ed. A. V. Filippenko (San Francisco: ASP), 123-136

Vestrand, W. T., Borozdin, K. N., Brumby, S. P., Casperson, D. E., Fenimore, E. E., Galassi, M. C., McGowan, K., Perkins, S. J., et al. 2002, Proc. SPIE, 4845, 126

Zacharias, N., Finch, C., Girard, T., Hambly, N., Wycoff, G., Zacharias, M. I., Castillo, D., Corbin, T., et al. 2010, AJ, 139, 2184 